\title{
18. UPPER QUATERNARY WESTERN ATLANTIC PALEOCEANOGRAPHY AND TERRIGENOUS SEDIMENTATION ON THE AMAZON FAN: A VIEW FROM STABLE ISOTOPES OF PLANKTONIC FORAMINIFERS AND BULK ORGANIC MATTER ${ }^{1}$
}

\author{
R.R. Schneider, ${ }^{2}$ P.J. Müller, ${ }^{2}$ B. Schlünz, ${ }^{2}$ M. Segl, ${ }^{2}$ W.J. Showers,${ }^{3}$ and G. Wefer ${ }^{2}$
}

\begin{abstract}
Three holes of 200 to $300 \mathrm{~m}$ in length (Holes 938A, 940A, and 942A) drilled during Ocean Drilling Program Leg 155 on the Amazon Fan have provided very expanded hemipelagic sediment records from crests of abandoned channel-levee systems. These sediments were analyzed for total organic and inorganic carbon contents, the $\delta^{13} \mathrm{C}$ ratios of total organic carbon, as well as $\delta^{18} \mathrm{O}$ and $\delta^{13} \mathrm{C}$ ratios of planktonic foraminifers, to evaluate western Atlantic paleocirculation and fan sedimentation processes with respect to upper Quaternary climate and sea-level change.

The foraminifer isotope records match the well-known pattern observed in cores from the open ocean and thus allow confirmation of preliminary shipboard bio- and magneto-stratigraphic chronologies. Isotopic excursions detected in the planktonic records in relatively undisturbed sediment sections can be related to similar events observed in the Southern Ocean, off southwest Africa, and in the Caribbean Sea. This indicates that changes in North Brazil Current hydrography off the Amazon River are closely connected to fluctuations in oceanwide surface circulation. There is no clear evidence for freshwater discharge events in the isotope records.

Organic and inorganic chemistry confirm the previous models of fan sedimentation for the last 140 k.y. Only during the maximum sea-level highstands was fan sedimentation dominated by fluxes of marine calcium carbonate and organic matter. During lowstands, below a threshold sea-level fall of 40 to $50 \mathrm{~m}$, terrigenous input dominated the sedimentation via distributary channel-levee systems, with fan growth occurring at very high rates ( $\sim 5$ to $20 \mathrm{~m} / \mathrm{k} . \mathrm{y}$.). Consistently low $\delta^{13} \mathrm{C}$ values of $\sim 1 \%$ o$27 \%$ for the organic matter in all glacial deposits suggest that the Amazon River load was transported directly to the upper and middle fan during sea-level lowstands, and not partially westward with the North Brazil Current as is the case of the modern ocean.
\end{abstract}

\section{INTRODUCTION}

\section{Objectives}

During Ocean Drilling Program (ODP) Leg 155, 34 holes at 17 sites were drilled on the Amazon Fan. The retrieved upper Quaternary sediments were expected to record fan growth patterns and changes in overlying oceanic circulation and river discharge in relation to sea-level and climate fluctuations. Based on shipboard biostratigraphic, paleomagnetic, and sedimentological investigations, a preliminary fan chronology for the last 250 k.y. was established (Flood, Piper, Klaus, et al., 1995), and it became obvious that it would be possible to assign different fan lithologies and their spatial distribution to distinct climatic periods and states of sea level.

Previous investigations on short piston and gravity cores $(\sim 10 \mathrm{~m}$ in length) documented that, during the transition from the last glacial maximum (LGM) to the Holocene, a dramatic change in sediment composition occurred all over the Amazon Fan. Damuth and Kumar (1975) described this change as a succession from dark gray greenish terrigenous muds characterizing glacial sedimentation into brownish carbonate-rich pelagic clays of Holocene age. The same pattern was found in the uppermost meter in every hole drilled during Leg 155 (Flood, Piper, Klaus, et al., 1995). The Holocene and older interglacial sea-level highstands are recorded by pelagic calcareous clays that were deposited across the entire fan at sedimentation rates of $\sim 0.02-$ $0.10 \mathrm{~m} / \mathrm{k}$.y., whereas deposition of terrigenous sediments took place on the continental shelf. During glacial sea-level lowstands, sedimen-

${ }^{1}$ Flood, R.D., Piper, D.J.W., Klaus, A., and Peterson, L.C. (Eds.), 1997. Proc. ODP, Sci. Results, 155: College Station, TX (Ocean Drilling Program).

${ }^{2}$ Fachbereich Geowissenschaften, Universitaet Bremen, 28334 Bremen, Federal Republic of Germany. rschneid@zfn.uni-bremen.de

${ }^{3}$ Department of Marine, Earth, and Atmospheric Sciences, North Carolina State University, Raleigh, NC 27695, U.S.A. tation of terrigenous clays, silts, and sands prevailed, distributed by several turbidite channel systems over the upper and lower fan (Manley and Flood, 1988; Fig. 1). Drilling of levee sequences associated with distributary channels showed that levees built by active channels accumulated at rates of 5-20 m/k.y., in contrast to the bioturbated hemipelagic muds that cover the levee crests of abandoned channels at rates of $1-3 \mathrm{~m} / \mathrm{k} . \mathrm{y}$.

From the short sediment cores previously recovered, it was known that this change in sediment source is documented not only in the organic matter vs. calcium carbonate content, but also in the stable carbon isotopic composition of bulk organic matter $\left(\delta^{13} C_{\text {org }}\right)$ all over the fan (Showers and Bevis, 1988; P.J. Müller, unpubl. data). Holocene sediments are characterized by low total organic carbon (TOC) contents with carbon isotope ratios near $-20 \%$, whereas sediments from the LGM contain higher amounts of TOC with isotope ratios about $-27 \%$. The isotope ratios thus, on average, represent the classical end-member values typical for marine-produced and terrestrial organic carbon, respectively (e.g., Fontugne and Duplessy, 1986). The carbonate contents vary inversely to the TOC contents, with very low carbonate concentrations in LGM terrigenous sediments and higher concentrations in the more pelagic Holocene sediments. Extending the short records obtained by gravity or piston coring, the cores drilled during ODP Leg 155 provided the opportunity to study whether this simple model of two different sediment sources was valid for longer than the LGM to Holocene period. Records of geochemical and isotopic proxies, several hundred meters in length, document the input of terrigenous organic matter vs. pelagic fluxes of marine organic carbon and carbonate and may help to elucidate the pattern of fan growth in correlation to global climate change and sea level over more than the last $20 \mathrm{k} . \mathrm{y}$.

Even though the Amazon Fan is mostly built up of very rapidly deposited and reworked sediments, it was expected that hemipelagic sediment sequences from levee crests of abandoned channel systems 
would allow paleoceanographic reconstructions of western Atlantic surface waters at a time resolution much higher than was obtained from the pelagic sediment records off Northeastern Brazil (e.g., Curry, Shackleton, Richter, et al., 1995; Ceara Rise, Leg 154). From the pre-Leg 155 studies on short cores, it was also clear that the main identifiable contribution from pelagic marine sources to the glacial fan sediments would be planktonic foraminifers. The foraminifers are thus the primary tool to relate fan growth and sedimentary facies to global climate change using their stable isotope ratios and faunal composition (see Showers et al. and Maslin et al., this volume). We investigated the $\delta^{18} \mathrm{O}$ and $\delta^{13} \mathrm{C}$ signals from planktonic foraminifers to find evidence for upper Quaternary changes in western tropical Atlantic surface waters, which may be related to fluctuations in river discharge and/or cross-equatorial surface circulation in conjunction with the North Brazil Current (NBC).

\section{Selected Sites}

Organic carbon and carbonate weight percentages (wt $\%), \delta^{13} \mathrm{C}_{\text {org }}$ values, and planktonic foraminifer $\delta^{18} \mathrm{O}$ and $\delta^{13} \mathrm{C}$ values were determined for the sediments retrieved from Holes 938A, 940A, and 942A. The selected sites represent areas of the upper eastern and central fan, as well as the western middle fan (Fig. 1). These sites were chosen because they were recovered from ancient channel-levee complexes previously mapped (Damuth et al., 1988) and, according to shipboard studies, comprise a wide range of levee sedimentation rates (Flood, Piper, Klaus, et al., 1995). Only these channel-levee sediments were thought to provide more or less undisturbed and turbidite-free sediment records.

Site 942 from the crest of an abandoned levee was intended to provide a hemipelagic reference section on the western part of the fan to study Atlantic paleoclimate and paleocirculation patterns. Of the sites selected, levee sedimentation rates at Site 942 were lowest; the upper $70 \mathrm{~m}$ of Hole $942 \mathrm{~A}$ probably spanning the last $130 \mathrm{k} . \mathrm{y}$. Below that core depth, levee sedimentation rates were much higher and more similar to those from the central and eastern channels of the Amazon Fan. At the upper eastern fan, Site 938 was chosen, located on the flank of the western levee of an abandoned channel-levee system from the LGM. The 310-m-long sediment record cored in Hole 938A probably covers the last 50 to $60 \mathrm{k} . \mathrm{y}$. and was expected to provide a very expanded, but still hemipelagic, paleoclimatic record. A less hemipelagic record, with the highest sedimentation rates and containing the transition from the LGM to the Holocene, was expected from Site 940. Located on the flank of the eastern levee of the youngest channel-levee system, the Amazon Channel (Fig. 1), Site 940 was chosen to understand levee growth with respect to sea-level rise from a glacial to an interglacial. The sediment record does not reach sediments older than $40 \mathrm{ka}$ at 209 meters below sea floor (mbsf), the bottom of Hole 940A.

\section{METHODS}

\section{Foraminifer Stable Isotopes}

All samples were selected from pelagic carbonate-rich muds and hemipelagic clayey silts or silty clays, whereas sandy or silty turbidites were avoided during sampling. Foraminifer samples were taken at depth intervals of roughly $50 \mathrm{~cm}$ at Sites 938 and 942, whereas at Site 940 , sample spacing is about every $1.50 \mathrm{~m}$. Inspection of wetsieved samples (>150- $\mu \mathrm{m}$ mesh size) revealed that only the upper 70 $\mathrm{m}$ of sediment from Hole $942 \mathrm{~A}$ and the Holocene sections from Holes 938A and 940A contained sufficient amounts of foraminifers for isotope analysis on single species from each sample. To enhance the number of data points for glacial sediments from Hole 938A, foraminifers out of two or three neighboring samples were combined to

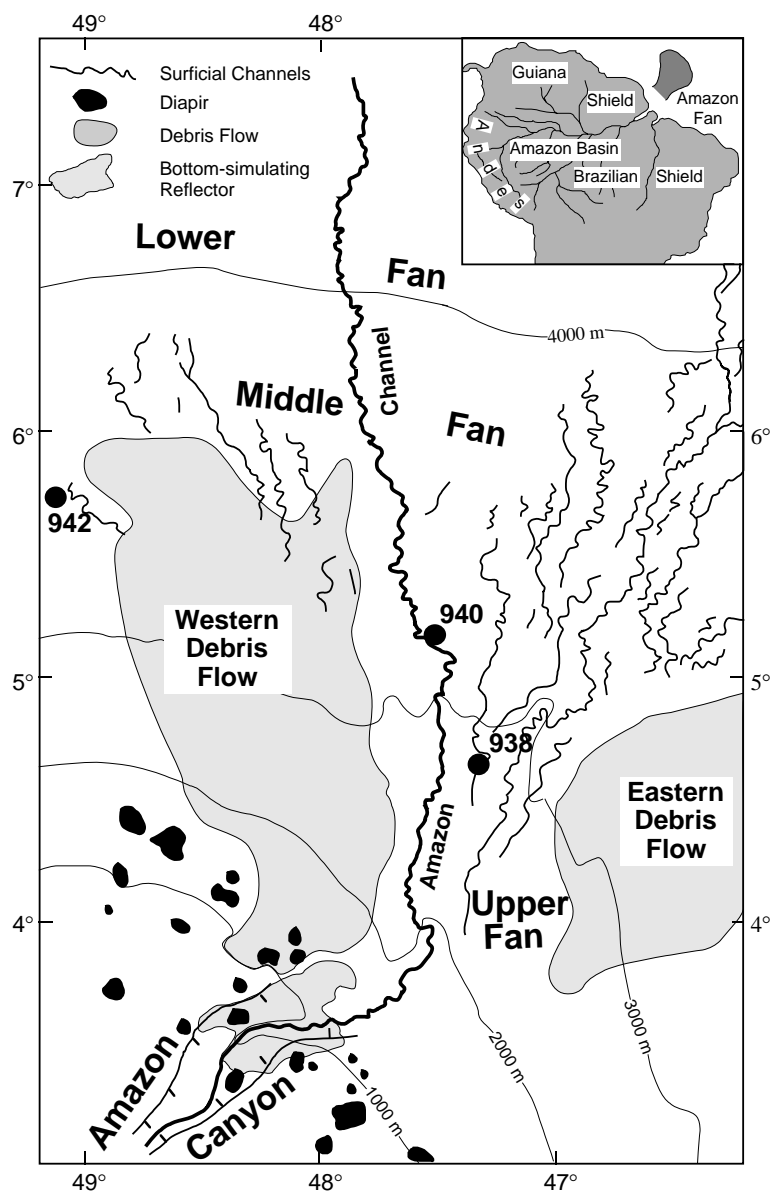

Figure 1. Location map showing the Leg 155 site positions in relation to morphologic features of the Amazon Fan. Modified from Flood, et. al., 1995; modified from Damuth et al., 1988, and Manley and Flood, 1988.

obtain the amount of $\mathrm{CaCO}_{3}$ sufficient for isotope analysis. The stable isotope data of combined samples are reported with respect to core depths that were linearly averaged from individual sample depths (see Table 1). For Site 940, affected by a very high terrigenous dilution, only the core catcher samples with a wet volume of more than $50 \mathrm{~cm}^{3}$ provided enough tests from an individual foraminifer species for stable isotope analysis.

Stable oxygen and carbon isotope ratios of the planktonic foraminifer Globigerinoides ruber (pink) were measured for Sites 938 and 940 using a FINNIGAN MAT 251 and an automated Kiel carbonate preparation line at Bremen University (e.g., Schneider et al., 1994). For Site 942, isotope data are from Globigerinoides sacculifer measured at North Carolina State University (Raleigh) with a similar technique. Calibration of the lab internal standard gas to the PDB standard scale was achieved using NBS (National Bureau of Standards, Wien) 18,19 , and $20 \mathrm{CaCO}_{3}$ standards. The analytical precision $( \pm 1 \sigma)$ based on replicate analyses of an internal $\mathrm{CaCO}_{3}$ lab standard is better than $0.07 \%$ and $0.05 \%$ for $\delta^{18} \mathrm{O}$ and $\delta^{13} \mathrm{C}$, respectively. The isotope data are listed in Table 1 with the exception of the samples from Site 942 that are reported in Showers et al. (this volume).

\section{Organic Carbon, Carbonate, and $\delta^{13} C_{o r g}$}

In core sections from Hole 942A, samples of $10 \mathrm{~cm}^{3}$ were taken for organic and inorganic carbon chemistry at depth increments of 
Table 1. Stable oxygen and carbon isotope ratios of $G$ ruber. in samples from Holes 938A and 940A.

\begin{tabular}{|c|c|c|c|c|c|c|c|c|c|c|c|}
\hline \multicolumn{3}{|c|}{ Hole 938A } & \multicolumn{3}{|c|}{ Hole 938A } & \multicolumn{3}{|c|}{ Hole $938 \mathrm{~A}$} & \multicolumn{3}{|c|}{ Hole $940 \mathrm{~A}$} \\
\hline $\begin{array}{l}\text { Depth } \\
\text { (mbsf) }\end{array}$ & $\delta^{18} \mathrm{O}(\% o)$ & $\delta^{13} \mathrm{C}(\% \circ)$ & $\begin{array}{l}\text { Depth } \\
\text { (mbsf) }\end{array}$ & $\delta^{18} \mathrm{O}(\% \circ)$ & $\delta^{13} \mathrm{C}(\% \circ)$ & $\begin{array}{l}\text { Depth } \\
\text { (mbsf) }\end{array}$ & $\delta^{18} \mathrm{O}(\% o)$ & $\delta^{13} \mathrm{C}(\% \circ)$ & $\begin{array}{l}\text { Depth } \\
\text { (mbsf) }\end{array}$ & $\delta^{18} \mathrm{O}(\% o)$ & $\delta^{13} \mathrm{C}(\% o)$ \\
\hline 0.23 & -1.84 & 1.97 & 68.30 & -0.36 & 1.46 & 142.40 & -0.83 & 1.23 & 0.13 & -1.99 & 1.69 \\
\hline 0.70 & -1.46 & 1.73 & 69.30 & -0.39 & 0.84 & 142.90 & -0.95 & 1.43 & 0.5 & -1.03 & 1.11 \\
\hline 1.20 & -1.57 & 1.68 & 71.15 & -0.42 & 1.18 & 143.40 & -1.00 & 1.40 & 1 & -0.85 & 0.76 \\
\hline 1.73 & -1.08 & 1.44 & 71.64 & -0.10 & 1.03 & 143.90 & -1.05 & 1.22 & 1.95 & -0.87 & 0.78 \\
\hline 2.20 & -1.15 & 0.78 & 72.15 & -0.27 & 0.47 & 146.16 & -0.52 & 0.86 & 2.46 & -0.98 & 0.17 \\
\hline 2.70 & -1.62 & 1.46 & 72.94 & -0.49 & 1.01 & 147.55 & -0.89 & 0.47 & 2.75 & -1.26 & 1.26 \\
\hline 3.23 & -0.91 & 0.53 & 73.48 & -0.43 & 0.81 & 148.80 & -0.67 & 0.79 & 1.3 & -0.59 & 0.48 \\
\hline 3.70 & -0.84 & 0.35 & 74.36 & -0.48 & 0.91 & 148.99 & -0.48 & 0.56 & 31.48 & -0.75 & 0.91 \\
\hline 4.20 & -0.81 & 1.00 & 75.07 & -0.47 & 0.65 & 153.76 & -0.72 & 0.96 & 39.7 & -0.39 & -0.15 \\
\hline 4.73 & -0.56 & -0.10 & 76.39 & -0.53 & 0.57 & 154.00 & -0.79 & 0.75 & 50.69 & -0.6 & 0.48 \\
\hline 5.20 & -0.31 & -0.52 & 77.09 & -0.32 & 0.81 & 154.50 & -0.95 & 0.90 & 58.71 & -0.2 & 0.49 \\
\hline 5.97 & -0.27 & -0.40 & 77.80 & -0.53 & 0.60 & 155.00 & -0.82 & 0.60 & 68.52 & -0.54 & 0.35 \\
\hline 6.92 & -0.65 & 0.43 & 78.84 & -0.33 & 1.25 & 155.30 & -0.92 & 1.27 & 77.01 & -0.76 & 0.69 \\
\hline 8.05 & -0.25 & -0.16 & 79.34 & -0.45 & 0.66 & 155.65 & -0.85 & 1.09 & 84.5 & -0.21 & 0.54 \\
\hline 11.05 & -0.88 & -0.55 & 79.79 & -0.60 & 0.46 & 160.56 & -0.76 & 0.95 & 93.24 & -0.05 & -0.02 \\
\hline 13.33 & -0.22 & 0.16 & 80.59 & -0.54 & 0.62 & 161.32 & -0.65 & 0.97 & 110.83 & -0.15 & 0.87 \\
\hline 15.63 & -0.33 & 0.55 & 81.25 & -0.25 & 0.75 & 162.03 & -0.73 & 1.06 & 120.55 & -0.18 & 0.25 \\
\hline 16.80 & 0.02 & 0.39 & 82.09 & -0.49 & 0.81 & 163.06 & -0.77 & 1.07 & 129.96 & -0.11 & 0.3 \\
\hline 16.85 & -0.07 & 0.63 & 82.94 & -0.32 & 0.81 & 164.83 & -0.45 & 1.17 & 149.33 & -0.34 & 0.1 \\
\hline 18.72 & -0.11 & 0.59 & 83.70 & -0.30 & 0.87 & 172.59 & -0.78 & 0.16 & 160.37 & -0.03 & 0.23 \\
\hline 19.72 & -0.07 & 0.16 & 84.30 & -0.49 & 1.07 & 174.79 & -0.63 & 0.60 & 167.5 & 0.12 & 0.43 \\
\hline 20.82 & 0.07 & 0.70 & 84.80 & -0.48 & 0.57 & 181.44 & -0.35 & 0.09 & 172.26 & -0.19 & 0.43 \\
\hline 22.23 & 0.08 & 0.93 & 85.20 & -0.49 & 0.83 & 182.74 & -0.32 & 0.49 & 187 & 0.02 & 0.45 \\
\hline 24.48 & -0.36 & 1.26 & 85.87 & -0.19 & -0.02 & 185.96 & -0.59 & 0.96 & 209.53 & -0.17 & 0.15 \\
\hline 26.69 & -0.19 & 1.00 & 86.71 & -0.32 & 0.59 & 187.13 & -0.53 & 0.23 & 217.77 & -0.06 & 0.44 \\
\hline 26.91 & 0.01 & 0.85 & 87.34 & -0.52 & 1.03 & 189.50 & -0.35 & 0.68 & 224.28 & -0.26 & 0.66 \\
\hline 28.04 & -0.24 & 0.94 & 87.97 & -0.22 & 0.17 & 195.34 & -0.44 & 0.82 & 236.14 & -0.06 & 0.62 \\
\hline 28.59 & -0.30 & 0.84 & 88.66 & -0.50 & 1.44 & 201.77 & -0.60 & 0.72 & 247.93 & -0.23 & 0.89 \\
\hline 29.22 & -0.35 & 0.15 & 89.71 & -0.35 & 0.80 & 203.25 & -0.50 & 0.93 & & & \\
\hline 29.88 & -0.26 & 0.52 & 90.14 & -0.58 & 0.77 & 204.95 & -0.26 & 0.48 & & & \\
\hline 30.04 & -0.11 & 0.88 & 90.74 & -0.67 & 1.18 & 206.17 & -0.47 & 0.51 & & & \\
\hline 30.69 & -0.27 & 0.68 & 91.22 & -0.60 & 0.49 & 206.69 & -0.44 & 0.94 & & & \\
\hline 31.39 & -0.31 & 0.06 & 92.13 & -0.53 & 0.97 & 207.74 & -0.26 & 0.68 & & & \\
\hline 31.54 & -0.27 & 0.64 & 93.83 & -0.68 & 0.57 & 214.30 & -0.37 & 0.36 & & & \\
\hline 32.31 & -0.42 & 1.04 & 94.36 & -0.42 & 0.07 & 216.30 & -0.58 & 0.53 & & & \\
\hline 32.76 & -0.45 & 0.87 & 94.77 & -0.18 & 0.41 & 220.53 & -0.44 & 0.30 & & & \\
\hline 33.04 & -0.10 & 0.56 & 95.27 & -0.41 & 0.42 & 222.13 & -0.25 & -0.24 & & & \\
\hline 33.61 & -0.30 & 0.81 & 95.86 & -0.52 & 0.86 & 224.87 & -0.47 & 0.59 & & & \\
\hline 34.18 & -0.34 & 1.18 & 96.52 & -0.44 & 0.39 & 231.84 & -0.40 & 0.24 & & & \\
\hline 34.54 & -0.11 & 0.09 & 98.02 & -0.43 & 0.62 & 234.23 & -0.53 & .77 & & & \\
\hline 35.21 & -0.12 & 0.24 & 98.96 & -0.53 & 0.59 & 242.97 & -0.59 & 1.10 & & & \\
\hline 35.71 & -0.16 & 0.68 & 102.52 & -0.10 & 0.48 & 244.47 & -0.54 & 1.12 & & & \\
\hline 37.05 & -0.10 & 0.73 & 106.16 & -0.86 & 0.28 & 245.99 & -0.63 & 0.83 & & & \\
\hline 38.26 & -0.21 & 0.62 & 110.27 & -0.27 & 0.85 & 247.25 & -0.54 & 0.70 & & & \\
\hline 40.63 & -0.11 & 0.86 & 118.07 & -0.70 & 0.59 & 249.01 & -0.54 & 1.18 & & & \\
\hline 41.60 & -0.04 & 0.34 & 118.69 & 0.61 & 0.96 & 250.27 & -0.68 & 0.83 & & & \\
\hline 42.10 & -0.26 & 0.72 & 119.57 & -0.60 & 0.41 & 250.80 & -0.58 & 1.18 & & & \\
\hline 42.60 & -0.15 & 1.03 & 122.44 & -0.19 & 0.40 & 255.85 & -0.68 & 0.84 & & & \\
\hline 43.98 & -0.11 & 1.13 & 123.46 & -0.28 & 0.17 & 257.11 & -0.65 & 0.52 & & & \\
\hline 45.78 & -0.04 & 0.95 & 123.72 & -0.54 & -0.09 & 257.90 & -0.32 & 0.41 & & & \\
\hline 46.56 & -0.15 & 0.55 & 124.67 & -0.28 & 0.43 & 260.57 & -0.48 & 1.04 & & & \\
\hline 49.04 & -0.35 & 0.88 & 126.20 & -0.43 & 0.43 & 266.22 & -0.60 & 0.92 & & & \\
\hline 52.56 & -0.34 & 0.70 & 126.72 & -0.27 & -0.10 & 269.01 & -0.44 & 0.68 & & & \\
\hline 54.03 & -0.36 & 0.78 & 127.18 & -0.05 & 0.05 & 270.23 & -0.54 & 0.62 & & & \\
\hline 55.22 & -0.31 & 0.46 & 130.60 & 0.15 & -0.59 & 274.64 & -0.60 & 0.73 & & & \\
\hline 56.41 & -0.48 & 0.86 & 132.75 & -0.18 & -0.27 & 284.70 & -0.70 & 0.50 & & & \\
\hline 59.45 & 0.01 & 1.19 & 135.05 & 0.04 & -0.74 & 288.62 & -0.34 & 0.41 & & & \\
\hline 63.83 & 0.01 & 0.58 & 136.60 & -0.33 & 0.52 & 293.35 & -0.23 & 1.13 & & & \\
\hline 64.80 & -0.32 & 1.24 & 137.91 & -0.35 & 0.71 & 297.40 & -0.21 & 0.37 & & & \\
\hline 64.99 & -0.09 & 1.14 & 138.46 & -0.50 & 0.87 & 301.95 & -0.42 & 1.30 & & & \\
\hline 66.30 & -0.41 & 1.10 & 138.54 & -0.51 & 0.54 & 305.26 & -0.06 & 1.50 & & & \\
\hline 67.29 & -0.20 & 0.83 & 141.40 & -0.79 & 0.84 & 306.13 & -0.33 & 1.07 & & & \\
\hline 67.80 & -0.36 & 1.17 & 141.90 & -0.97 & 1.41 & & & & & & \\
\hline
\end{tabular}

Notes: Data for Hole 938A are reported vs. average core depth (mbsf) according to a combination of individual samples for isotope analyses (see Methods). Data for Hole 940A only compose core catcher samples. All values are given in per mil (\%o) vs. PDB.

$\sim 50 \mathrm{~cm}$, whereas from Hole 938A, they were taken at increments of $\sim 150 \mathrm{~cm}$. Sampling on sediment sections from Hole 940A was more irregular according to the distribution of hemipelagic and turbiditic intervals. At the latter site, $\delta^{13} \mathrm{C}_{\text {org }}$ measurements were carried out only on shipboard carbonate samples.

Total carbon (TC) and TOC values were obtained by combustion at $1050^{\circ} \mathrm{C}$, using a HERAEUS CHN-O-Rapid elemental analyzer, following standard procedures described in Müller et al. (1993). The carbonate content of the samples was calculated from the difference between TC and TOC and expressed as calcite $\left[\mathrm{CaCO}_{3}=8.33 \cdot(\mathrm{TC}\right.$ - TOC)]. TOC and $\mathrm{CaCO}_{3}$ values are reported in percent dry weight, not corrected for the salt content. The relative precision of the mea- surements, based on duplicates and control analysis of a lab internal reference sediment sample (WST2), was better than 3\%.

The stable carbon isotope composition of TOC $\left(\delta^{13} \mathrm{C}_{\text {org }}\right)$ was determined with a FINNIGAN MAT delta E mass spectrometer and a HERAEUS CHN-Rapid elemental analyzer interfaced by an automated trapping box. Sample preparation was equivalent to that performed for TOC analyses (Müller et al., 1993). The overall analytical precision $( \pm 1 \sigma$, based on duplicates and repeated analyses of the labinternal WS1 standard) was better than $0.1 \%$. The $\delta^{13} C_{\text {org }}$ values are reported in standard notation relative to $\mathrm{PDB}$. All data for TOC and carbonate content, as well as for $\delta^{13} C_{\text {org }}$, are listed in Table 2 with respect to sample depths in mbsf. 
Table 2. Total organic (TOC) and inorganic carbon $\left(\mathrm{CaCO}_{3}\right)$ contents, and $\delta^{13} \mathrm{C}_{\text {org }}$ values from Holes $938 \mathrm{~A}$, $940 \mathrm{~A}$, and 942A reported vs. corrected depths.

\begin{tabular}{|c|c|c|c|c|c|c|c|c|c|}
\hline & & Iole 938A & & & & & & ole 942 B & \\
\hline $\begin{array}{l}\text { Core, section, } \\
\text { interval }(\mathrm{cm})\end{array}$ & $\begin{array}{l}\text { Depth } \\
\text { (mbsf) }\end{array}$ & Remarks & TOC & $\mathrm{CaCO}_{3}$ & $\mathrm{~d}^{13} \mathrm{C}_{\text {org }}$ & $\begin{array}{l}\text { Core, section, } \\
\text { interval }(\mathrm{cm})\end{array}$ & $\begin{array}{l}\text { Depth } \\
\text { (mbsf) }\end{array}$ & Remarks & TOC \\
\hline $1 \mathrm{H}-1,8-9$ & 0.08 & Ship & 0.52 & 31.5 & -20.05 & $1 \mathrm{H}-1,50-56$ & 0.5 & $942 \mathrm{C}$ & 0.32 \\
\hline $1 \mathrm{H}-1,20-22$ & 0.20 & & 0.43 & 33.8 & -20.47 & $1 \mathrm{H}-1,83-88$ & 0.83 & $942 \mathrm{C}$ & 0.51 \\
\hline $1 \mathrm{H}-1,68-70$ & 0.68 & & 0.45 & 21.2 & -21.59 & $1 \mathrm{H}-1,92-94$ & 0.92 & & 0.68 \\
\hline $1 \mathrm{H}-1,118-120$ & 1.18 & & 0.66 & 2.8 & -24.35 & $1 \mathrm{H}-1,137-139$ & 1.37 & & 0.85 \\
\hline $1 \mathrm{H}-2,20-22$ & 1.70 & & 0.69 & 1.2 & -25.63 & $1 \mathrm{H}-2,6-7$ & 1.56 & Ship & 0.81 \\
\hline $1 \mathrm{H}-2,68-70$ & 2.18 & & 0.9 & 0.2 & -27.12 & $1 \mathrm{H}-2,31-33$ & 1.81 & & 0.81 \\
\hline $1 \mathrm{H}-2,118-120$ & 2.68 & & 0.86 & 0.4 & -27.17 & $1 \mathrm{H}-2,81-83$ & 2.31 & & 0.78 \\
\hline $1 \mathrm{H}-3,20-22$ & 3.20 & & 0.86 & 1 & -27.13 & $1 \mathrm{H}-2,131-133$ & 2.81 & & 0.92 \\
\hline $1 \mathrm{H}-3,68-70$ & 3.68 & & 0.92 & 0.9 & -27.2 & $1 \mathrm{H}-3,16-18$ & 3.16 & & 0.96 \\
\hline $1 \mathrm{H}-3,118-120$ & 4.18 & & 0.95 & 1.1 & -27.36 & $2 \mathrm{H}-1,25-27$ & 4.05 & & 0.91 \\
\hline $1 \mathrm{H}-4,20-22$ & 4.70 & & 0.95 & 1.2 & -27.38 & $2 \mathrm{H}-1,74-76$ & 4.54 & & 0.81 \\
\hline $1 \mathrm{H}-4,40-41$ & 4.90 & Ship & 0.97 & 0.8 & -32 & $2 \mathrm{H}-1,124-126$ & 5.04 & & 0.71 \\
\hline $1 \mathrm{H}-4,68-70$ & 5.18 & & 0.95 & 1.2 & -27.49 & $2 \mathrm{H}-2,24-26$ & 5.54 & & 0.80 \\
\hline $1 \mathrm{H}-4,118-120$ & 5.68 & & 0.97 & 0.7 & -27.38 & $2 \mathrm{H}-2,74-76$ & 6.04 & & 0.65 \\
\hline $1 \mathrm{H}-5,20-22$ & 6.20 & & 0.94 & 0.6 & -27.27 & $2 \mathrm{H}-2,124-126$ & 6.54 & & 0.59 \\
\hline $1 \mathrm{H}-5,60-63$ & 6.60 & & 0.93 & 0.5 & -27.24 & $2 \mathrm{H}-3,24-26$ & 7.04 & & 0.62 \\
\hline $1 \mathrm{H}-5,118-120$ & 7.18 & & 0.96 & 0.1 & -27.14 & $2 \mathrm{H}-3,73-75$ & 7.53 & & 0.70 \\
\hline $2 \mathrm{H}-1,25-27$ & 7.83 & & 0.92 & 1.2 & -27.12 & $2 \mathrm{H}-3,124-126$ & 8.04 & & 0.67 \\
\hline $2 \mathrm{H}-1,75-77$ & 8.29 & & 0.94 & 1 & -27.17 & $2 \mathrm{H}-4,24-26$ & 8.54 & & 0.62 \\
\hline $2 \mathrm{H}-1,126-128$ & 8.77 & & 0.9 & 1.4 & -27.06 & $2 \mathrm{H}-4,37-38$ & 8.67 & Ship & 0.78 \\
\hline $2 \mathrm{H}-2,25-27$ & 9.22 & & 0.87 & 1.4 & -26.97 & $2 \mathrm{H}-4,74-76$ & 9.04 & & 0.79 \\
\hline $2 \mathrm{H}-2,36-37$ & 9.32 & Ship & 0.7 & 4.3 & -26.77 & $2 \mathrm{H}-4,124-126$ & 9.54 & & 0.76 \\
\hline $2 \mathrm{H}-2,76-78$ & 9.69 & & 0.81 & 1.7 & -26.77 & $2 \mathrm{H}-5,24-26$ & 10.04 & & 0.62 \\
\hline $2 \mathrm{H}-2,126-128$ & 10.15 & & 0.8 & 1 & -26.7 & $2 \mathrm{H}-5,74-76$ & 10.54 & & 0.68 \\
\hline $2 \mathrm{H}-3,25-27$ & 10.60 & & 0.83 & 1.2 & -26.6 & $2 \mathrm{H}-5,124-126$ & 11.04 & & 0.64 \\
\hline $2 \mathrm{H}-3,72-74$ & 11.03 & & 0.79 & 8.6 & -26.45 & $2 \mathrm{H}-6,24-26$ & 11.54 & & 0.71 \\
\hline $2 \mathrm{H}-3,126-128$ & 11.54 & & 0.85 & 0.6 & -26.42 & $2 \mathrm{H}-6,75-77$ & 12.05 & & 0.63 \\
\hline $2 \mathrm{H}-4,25-27$ & 11.99 & & 0.98 & 1.2 & -27.11 & $3 \mathrm{H}-1,25-27$ & 13.54 & & 0.73 \\
\hline $2 \mathrm{H}-4,78-79$ & 12.48 & Ship & 0.99 & 1.6 & -26.9 & $3 \mathrm{H}-1,80-82$ & 14.08 & & 0.50 \\
\hline $2 \mathrm{H}-4,85-87$ & 12.55 & & 0.97 & 1.5 & -26.99 & $3 \mathrm{H}-1,125-127$ & 14.52 & & 0.73 \\
\hline $2 \mathrm{H}-4,126-128$ & 12.93 & & 1.01 & 0.7 & -27.04 & $3 \mathrm{H}-2,25-27$ & 15.01 & & 0.62 \\
\hline $2 \mathrm{H}-5,25-27$ & 13.38 & & 0.96 & 2.5 & -27.09 & $3 \mathrm{H}-2,75-77$ & 15.50 & & 0.71 \\
\hline $2 \mathrm{H}-5,81-83$ & 13.90 & & 1 & 1.2 & -27.02 & $3 \mathrm{H}-2,124-126$ & 15.97 & & 0.77 \\
\hline $2 \mathrm{H}-5,126-128$ & 14.31 & & 1 & 1.8 & -27.09 & $3 \mathrm{H}-3,25-27$ & 16.47 & & 0.73 \\
\hline $2 \mathrm{H}-6,25-27$ & 14.76 & & 0.94 & 1.2 & -27 & $3 \mathrm{H}-3,75-77$ & 16.96 & & 0.67 \\
\hline $2 \mathrm{H}-6,76-78$ & 15.24 & & 0.91 & 1.7 & -26.92 & $3 \mathrm{H}-3,124-126$ & 17.44 & & 0.82 \\
\hline $2 \mathrm{H}-6,126-128$ & 15.70 & & 0.92 & 2.2 & -26.96 & $3 \mathrm{H}-4,25-27$ & 17.93 & & 0.65 \\
\hline $2 \mathrm{H}-7,25-27$ & 16.15 & & 0.96 & 1.7 & -26.92 & $3 \mathrm{H}-4,36-37$ & 18.04 & Ship & 0.63 \\
\hline $2 \mathrm{H}-7,76-78$ & 16.62 & & 0.94 & 1.8 & -26.89 & $3 \mathrm{H}-4,75-77$ & 18.42 & & 0.74 \\
\hline $3 \mathrm{H}-1,26-28$ & 17.36 & & 0.99 & 1.7 & -26.81 & $3 \mathrm{H}-4,124-126$ & 18.90 & & 0.88 \\
\hline $3 \mathrm{H}-1,75-77$ & 17.85 & & 0.97 & 2.3 & -26.81 & $3 \mathrm{H}-5,25-27$ & 19.40 & & 0.74 \\
\hline $3 \mathrm{H}-1,126-128$ & 18.23 & & 0.82 & 2.7 & -26.84 & $3 \mathrm{H}-5,75-77$ & 19.88 & & 0.83 \\
\hline $3 \mathrm{H}-2,22-24$ & 18.80 & & 0.82 & 2.4 & -26.57 & $3 \mathrm{H}-5,124-126$ & 20.36 & & 0.76 \\
\hline $3 \mathrm{H}-2,78-80$ & 19.24 & & 0.99 & 1.6 & -26.57 & $3 \mathrm{H}-6,25-27$ & 20.86 & & 0.79 \\
\hline $3 \mathrm{H}-2,117-119$ & 19.61 & & 0.9 & 2 & -26.48 & $3 \mathrm{H}-6,73-75$ & 21.33 & & 0.76 \\
\hline $3 \mathrm{H}-3,26-28$ & 20.32 & & 0.98 & 1.7 & -26.55 & $3 \mathrm{H}-6,124-126$ & 21.83 & & 0.69 \\
\hline $3 \mathrm{H}-3,75-77$ & 20.74 & & 0.93 & 1.9 & -26.58 & $3 \mathrm{H}-7,25-27$ & 22.32 & & 0.70 \\
\hline $3 \mathrm{H}-3,129-131$ & 21.15 & & 0.92 & 2.3 & -26.68 & $4 \mathrm{H}-1,25-27$ & 23.04 & & 0.82 \\
\hline $3 \mathrm{H}-4,23-25$ & 21.78 & & 0.97 & 2.2 & -26.75 & $4 \mathrm{H}-1,80-92$ & 23.56 & & 0.55 \\
\hline $3 \mathrm{H}-4,57-58$ & 22.06 & Ship & 0.89 & 1.9 & -26.84 & $4 \mathrm{H}-1,130-132$ & 24.03 & & 0.73 \\
\hline $3 \mathrm{H}-4,76-78$ & 22.23 & & 0.93 & 1.6 & -26.52 & $4 \mathrm{H}-2,25-27$ & 24.45 & & 0.77 \\
\hline $3 \mathrm{H}-4,126-128$ & 22.63 & & 0.96 & 1.2 & -26.55 & $4 \mathrm{H}-2,74-76$ & 24.91 & & 0.72 \\
\hline $3 \mathrm{H}-5,25-27$ & 23.27 & & 0.96 & 2.1 & -26.47 & $4 \mathrm{H}-2,130-132$ & 25.44 & & 0.72 \\
\hline $3 \mathrm{H}-5,74-76$ & 23.73 & & 0.93 & 1.2 & -26.55 & $4 \mathrm{H}-3,33-35$ & 25.94 & & 0.77 \\
\hline $3 \mathrm{H}-5,91-92$ & 23.88 & Ship & 0.49 & 1.2 & -26.83 & $4 \mathrm{H}-3,69-70$ & 26.28 & Ship & 0.71 \\
\hline $3 \mathrm{H}-5,126-128$ & 24.21 & & 0.96 & 1.2 & -26.58 & $4 \mathrm{H}-3,79-81$ & 26.38 & & 0.62 \\
\hline $3 \mathrm{H}-6,26-28$ & 24.32 & & 0.87 & 2 & -26.29 & $4 \mathrm{H}-3,130-132$ & 26.86 & & 0.79 \\
\hline $3 \mathrm{H}-6,79-81$ & 24.81 & & 0.96 & 3.7 & -26.76 & $4 \mathrm{H}-4,3-4$ & 27.07 & Ship & 0.53 \\
\hline $3 \mathrm{H}-6,128-130$ & 25.27 & & 0.93 & 1.2 & -26.64 & $4 \mathrm{H}-4,25-27$ & 27.28 & & 0.47 \\
\hline $3 \mathrm{H}-7,23-25$ & 26.25 & & 0.93 & 1.7 & -26.45 & $4 \mathrm{H}-4,79-81$ & 27.79 & & 0.53 \\
\hline $3 \mathrm{H}-7,76-78$ & 26.71 & & 0.94 & 1.3 & -26.54 & $4 \mathrm{H}-4,130-132$ & 28.27 & & 0.44 \\
\hline $4 \mathrm{H}-2,15-17$ & 27.14 & & 0.87 & 2.2 & -26.79 & $4 \mathrm{H}-2,148-150$ & 28.65 & $942 B$ & 0.43 \\
\hline & & & & & & & & Ship & \\
\hline $4 \mathrm{H}-2,74-76$ & 27.67 & & 0.89 & 2.1 & -26.75 & $4 \mathrm{H}-5,28-30$ & 28.73 & & 0.51 \\
\hline $4 \mathrm{H}-2,142-144$ & 28.23 & & 0.92 & 3.3 & -26.77 & $4 \mathrm{H}-5,76-78$ & 29.18 & & 0.49 \\
\hline $4 \mathrm{H}-3,14-16$ & 28.59 & & 0.85 & 1.9 & -26.62 & $4 \mathrm{H}-5,130-132$ & 29.69 & & 0.47 \\
\hline $4 \mathrm{H}-3,77-79$ & 29.20 & & 0.85 & 1.8 & -26.75 & $4 \mathrm{H}-6,25-27$ & 30.11 & & 0.46 \\
\hline $4 \mathrm{H}-3,142-144$ & 29.67 & & 0.91 & 1.6 & -26.66 & $4 \mathrm{H}-6,79-81$ & 30.62 & & 0.48 \\
\hline $4 \mathrm{H}-4,13-15$ & 30.04 & & 0.96 & 1.4 & -26.8 & $4 \mathrm{H}-6,130-132$ & 31.10 & & 0.47 \\
\hline $4 \mathrm{H}-4,74-76$ & 30.51 & & 0.95 & 1.6 & -26.63 & $4 \mathrm{H}-7,25-27$ & 31.53 & & 0.56 \\
\hline $4 \mathrm{H}-4,117-118$ & 30.82 & Ship & 0.92 & 1.2 & -26.49 & $4 \mathrm{H}-7,75-77$ & 32.00 & & 0.49 \\
\hline $4 \mathrm{H}-4,144-146$ & 31.06 & & 0.96 & 1.4 & -26.68 & $5 \mathrm{H}-1,26-28$ & 32.56 & & 0.62 \\
\hline $4 \mathrm{H}-5,8-10$ & 31.44 & & 0.86 & 1.8 & -26.62 & $5 \mathrm{H}-1,47-49$ & 32.76 & Ship & 0.54 \\
\hline $4 \mathrm{H}-5,75-77$ & 32.01 & & 0.91 & 1.3 & -26.63 & $5 \mathrm{H}-1,75-77$ & 33.04 & & 0.76 \\
\hline $4 \mathrm{H}-5,130-132$ & 32.54 & & 0.94 & 1.5 & -26.74 & $5 \mathrm{H}-1,126-128$ & 33.54 & & 0.66 \\
\hline $4 \mathrm{H}-6,9-10$ & 32.91 & & 0.9 & 1.8 & -26.64 & $5 \mathrm{H}-2,40-42$ & 34.17 & & 0.61 \\
\hline $4 \mathrm{H}-6,66-68$ & 33.43 & & 0.87 & 1.7 & -26.7 & $5 \mathrm{H}-2,87-89$ & 34.64 & & 0.65 \\
\hline $4 \mathrm{H}-6,123-125$ & 33.86 & & 0.88 & 2.2 & -26.64 & $5 \mathrm{H}-2,132-134$ & 35.08 & & 0.59 \\
\hline $4 \mathrm{H}-7,9-11$ & 34.36 & & 0.94 & 1.2 & -26.69 & $5 \mathrm{H}-3,42-44$ & 35.67 & & 0.54 \\
\hline $4 \mathrm{H}-7,75-77$ & 34.96 & & 0.95 & 1.8 & -26.87 & $5 \mathrm{H}-3,121-123$ & 36.45 & & 0.59 \\
\hline $4 \mathrm{H}-7,126-128$ & 35.45 & & 0.96 & 1.5 & -26.63 & $5 \mathrm{H}-4,24-26$ & 36.72 & & 0.53 \\
\hline $4 \mathrm{H}-8,12-14$ & 35.85 & & 0.98 & 1.3 & -26.75 & $5 \mathrm{H}-4,99-101$ & 37.46 & & 0.53 \\
\hline $5 \mathrm{H}-3,25-28$ & 39.06 & & 0.9 & 0.5 & -26.34 & $5 \mathrm{H}-5,22-24$ & 38.18 & & 0.64 \\
\hline $5 \mathrm{H}-3,75-78$ & 39.41 & & 0.9 & 1.2 & -26.55 & $5 \mathrm{H}-6,33-35$ & 38.83 & & 0.60 \\
\hline $5 \mathrm{H}-3,138-140$ & 39.84 & & 1 & 1 & -26.57 & $5 \mathrm{H}-6,88-90$ & 39.37 & & 0.69 \\
\hline $5 \mathrm{H}-4,25-28$ & 40.52 & & 0.9 & 0.9 & -26.43 & $5 \mathrm{H}-6,125-127$ & 39.74 & & 0.58 \\
\hline $5 \mathrm{H}-4,45-46$ & 40.71 & Ship & 0.9 & 1.5 & -26.26 & $5 \mathrm{H}-7,20-22$ & 40.18 & & 0.55 \\
\hline
\end{tabular}


Table 2 (continued).

\begin{tabular}{|c|c|c|c|c|c|c|c|c|c|c|c|}
\hline & & Iole 938A & & & & & & Iole 942B & & & \\
\hline $\begin{array}{l}\text { Core, section, } \\
\text { interval }(\mathrm{cm})\end{array}$ & $\begin{array}{l}\text { Depth } \\
\text { (mbsf) }\end{array}$ & Remarks & TOC & $\mathrm{CaCO}_{3}$ & $\mathrm{~d}^{13} \mathrm{C}_{\mathrm{org}}$ & $\begin{array}{l}\text { Core, section, } \\
\text { interval }(\mathrm{cm})\end{array}$ & $\begin{array}{l}\text { Depth } \\
\text { (mbsf) }\end{array}$ & Remarks & TOC & $\mathrm{CaCO}_{3}$ & $\mathrm{~d}^{13} \mathrm{C}_{\mathrm{org}}$ \\
\hline $5 \mathrm{H}-4,72-75$ & 40.97 & & 0.9 & 1.7 & -26.47 & $5 \mathrm{H}-7,46-47$ & 40.44 & Ship & 0.54 & 8.4 & -22.84 \\
\hline $5 \mathrm{H}-4,126-128$ & 41.43 & & 0.9 & 1 & -26.52 & $5 \mathrm{H}-7,76-78$ & 40.74 & & 0.49 & 8.7 & -22.57 \\
\hline $5 \mathrm{H}-5,25-28$ & 41.97 & & 0.9 & 0.8 & -26.61 & $5 \mathrm{H}-7,123-125$ & 41.20 & & 0.54 & 11.4 & -22.04 \\
\hline $5 \mathrm{H}-5,75-78$ & 42.46 & & 0.9 & 1.2 & -26.67 & $6 \mathrm{H}-1,25-27$ & 42.05 & & 0.53 & 0.1 & -23.36 \\
\hline $5 \mathrm{H}-5,126-128$ & 42.95 & & 0.9 & 1.2 & -26.73 & $6 \mathrm{H}-1,46-47$ & 42.26 & Ship & 0.36 & 1.5 & -24.68 \\
\hline $5 \mathrm{H}-6,75-78$ & 43.39 & & 0.9 & 1.1 & -26.64 & $6 \mathrm{H}-1,75-77$ & 42.54 & & 0.51 & 0.3 & -25.12 \\
\hline $5 \mathrm{H}-6,22-25$ & 43.40 & & 0.9 & 1.2 & -26.66 & $6 \mathrm{H}-1,126-128$ & 43.05 & & 0.44 & 9.6 & -26.03 \\
\hline $5 \mathrm{H}-6,126-128$ & 44.24 & & 0.9 & 1.2 & -26.68 & $6 \mathrm{H}-2,26-28$ & 43.54 & & 0.32 & 0.9 & -26.27 \\
\hline $5 \mathrm{H}-7,28-31$ & 44.85 & & 0.9 & 1.9 & -26.61 & $6 \mathrm{H}-2,56-58$ & 43.84 & & 0.46 & 1.1 & -25.86 \\
\hline $5 \mathrm{H}-7,75-78$ & 45.30 & & 0.9 & 0.9 & -26.59 & $6 \mathrm{H}-3,17-19$ & 44.94 & & 0.40 & 2.0 & -26.44 \\
\hline $6 \mathrm{H}-2,25-27$ & 46.04 & & 0.9 & 1 & -26.4 & $6 \mathrm{H}-3,56-58$ & 45.33 & & 0.47 & 1.3 & -26.22 \\
\hline $6 \mathrm{H}-2,75-77$ & 46.49 & & 0.9 & 0.7 & -26.57 & $6 \mathrm{H}-4,145-147$ & 47.69 & & 0.49 & 1.6 & -26.12 \\
\hline $6 \mathrm{H}-2,126-128$ & 46.95 & & 0.9 & 1.1 & -26.65 & $6 \mathrm{H}-5,29-31$ & 48.03 & & 0.51 & 1.1 & -26.17 \\
\hline $6 \mathrm{H}-3,25-27$ & 47.39 & & 0.9 & 0.7 & -26.48 & H-5, 135-137 & 49.08 & & 0.41 & 1.2 & -26.20 \\
\hline $6 \mathrm{H}-3,75-77$ & 47.83 & & 0.9 & 0.9 & -26.73 & $6 \mathrm{H}-5,140-141$ & 49.13 & Ship & 0.35 & 2.0 & -25.74 \\
\hline $6 \mathrm{H}-3,124-126$ & 48.27 & & 0.9 & 0.7 & -26.36 & $6 \mathrm{H}-6,9-11$ & 49.32 & & 0.45 & 1.4 & -26.35 \\
\hline $6 \mathrm{H}-4,25-27$ & 48.73 & & 0.9 & 0.6 & -26.39 & $6 \mathrm{H}-6,81-83$ & 50.03 & & 0.47 & 1.4 & -26.71 \\
\hline $6 \mathrm{H}-4,75-77$ & 49.18 & & 0.9 & 0.6 & -26.28 & $6 \mathrm{H}-6,140-142$ & 50.62 & & 0.38 & 1.1 & -26.54 \\
\hline $6 \mathrm{H}-4,78-79$ & 49.21 & Ship & 0.8 & 0.9 & -26.3 & $6 \mathrm{H}-7,4-5$ & 50.76 & & 0.45 & 2.7 & -26.35 \\
\hline $6 \mathrm{H}-4,125-127$ & 49.63 & & 0.9 & 1.2 & -26.36 & $7 \mathrm{H}-1,21-23$ & 51.52 & & 0.50 & 1.1 & -25.84 \\
\hline $6 \mathrm{H}-4,134-135$ & 49.71 & Ship & 0.9 & 0.8 & -26.32 & $7 \mathrm{H}-1,118-120$ & 52.45 & & 0.49 & 1.0 & -26.50 \\
\hline $6 \mathrm{H}-5,25-27$ & 50.08 & & 0.9 & 0.7 & -26.43 & $7 \mathrm{H}-2,47-49$ & 53.22 & & 0.47 & 1.6 & -26.65 \\
\hline $6 \mathrm{H}-5,81-83$ & 50.58 & & 0.9 & 3.7 & -26.63 & $7 \mathrm{H}-2,114-116$ & 53.87 & & 0.47 & 1.9 & -26.66 \\
\hline $6 \mathrm{H}-5,125-127$ & 50.97 & & 0.8 & 1.2 & -26.53 & $7 \mathrm{H}-3,35-37$ & 54.56 & & 0.49 & 1.5 & -26.61 \\
\hline $6 \mathrm{H}-6,27-29$ & 51.44 & & 0.9 & 1.3 & -26.79 & $7 \mathrm{H}-3,109-111$ & 55.28 & & 0.46 & 1.1 & -26.18 \\
\hline $6 \mathrm{H}-6,75-77$ & 51.87 & & 0.9 & 1.7 & -26.63 & $7 \mathrm{H}-4,35-37$ & 56.00 & & 0.46 & 1.4 & -26.48 \\
\hline $6 \mathrm{H}-6,125-127$ & 52.32 & & 0.9 & 1.2 & -26.57 & $7 \mathrm{H}-3,109-111$ & 55.28 & & 0.46 & 1.1 & -26.18 \\
\hline $6 \mathrm{H}-7,25-27$ & 52.77 & & 0.9 & 1.5 & -26.66 & $7 \mathrm{H}-4,35-37$ & 56.00 & & 0.46 & 1.4 & -26.48 \\
\hline $6 \mathrm{H}-7,75-77$ & 53.22 & & 0.9 & 1.2 & -26.58 & $7 \mathrm{H}-4,80-82$ & 56.44 & & 0.45 & 1.1 & -26.42 \\
\hline $6 \mathrm{H}-7,125-127$ & 53.66 & & 0.9 & 1.4 & -26.67 & $7 \mathrm{H}-4,111-113$ & 56.74 & & 0.46 & 1.5 & -26.49 \\
\hline $6 \mathrm{H}-8,26-28$ & 54.12 & & 0.9 & 1.3 & -26.44 & $7 \mathrm{H}-4,115-116$ & 56.78 & Ship & 0.08 & 1.3 & \\
\hline $6 \mathrm{H}-8,74-76$ & 54.47 & & 0.9 & 1.7 & -26.76 & $7 \mathrm{H}-5,35-37$ & 57.49 & & 0.52 & 1.1 & -26.57 \\
\hline $7 \mathrm{H}-2,76-81$ & 56.25 & & 0.9 & 1 & -26.5 & $7 \mathrm{H}-5,142-144$ & 58.53 & & 0.43 & 1.1 & -26.66 \\
\hline $7 \mathrm{H}-3,76-81$ & 57.73 & & 0.9 & 1.2 & -26.46 & $7 \mathrm{H}-6,5-65$ & 8.65 & Ship & 0.36 & 2.2 & -26.09 \\
\hline $7 \mathrm{H}-4,53-54$ & 58.92 & Ship & 0.9 & 1.4 & -26.38 & $7 \mathrm{H}-6,24-26$ & 58.84 & & 0.52 & 0.9 & -26.27 \\
\hline $7 \mathrm{H}-4,76-81$ & 59.14 & & 0.9 & 2.2 & -26.57 & $7 \mathrm{H}-6,119-121$ & 59.76 & & 0.48 & 1.8 & -26.70 \\
\hline $7 \mathrm{H}-5,76-81$ & 60.70 & & 0.9 & 0.2 & -26.4 & $7 \mathrm{H}-7,42-44$ & 60.48 & & 0.52 & 1.7 & -26.59 \\
\hline $7 \mathrm{H}-5,84-85$ & 60.78 & Ship & 1 & 2.2 & -26.25 & $8 \mathrm{H}-1,24-26$ & 61.04 & & 0.54 & 1.8 & -26.64 \\
\hline $7 \mathrm{H}-7,76-81$ & 63.06 & & 0.9 & 1.2 & -26.46 & $8 \mathrm{H}-1,65-67$ & 61.45 & & 0.53 & 1.0 & -26.24 \\
\hline $8 \mathrm{H}-1,75-80$ & 65.33 & & 0.9 & 1.5 & -26.45 & $8 \mathrm{H}-1,125-127$ & 61.98 & & 0.55 & 1.2 & -26.71 \\
\hline $8 \mathrm{H}-2,75-80$ & 66.74 & & 0.9 & 1.3 & -26.56 & $8 \mathrm{H}-2,16-18$ & 62.46 & & 0.58 & 3.2 & -26.25 \\
\hline $8 \mathrm{H}-3,75-80$ & 68.27 & & 0.9 & 1 & -26.58 & $8 \mathrm{H}-2,71-73$ & 62.94 & & 0.51 & 1.2 & -26.21 \\
\hline $8 \mathrm{H}-4,75-80$ & 69.73 & & 0.9 & 2.4 & -26.88 & $8 \mathrm{H}-2,126-128$ & 63.49 & & 0.51 & 0.7 & -25.65 \\
\hline $8 \mathrm{H}-5,29-30$ & 70.60 & Ship & 0.9 & 0.7 & -26.26 & $8 \mathrm{H}-3,25-27$ & 64.05 & & 0.43 & 1.4 & -25.63 \\
\hline $8 \mathrm{H}-5,58-59$ & 70.84 & Ship & 1 & 1 & -26.77 & $8 \mathrm{H}-3,75-77$ & 64.55 & & 0.46 & 0.9 & -25.77 \\
\hline $8 \mathrm{H}-5,75-80$ & 71.00 & & 0.9 & 1.7 & -26.48 & $8 \mathrm{H}-3,125-127$ & 65.05 & & 0.60 & 1.5 & -23.89 \\
\hline $8 \mathrm{H}-6,75-80$ & 72.49 & & 1 & 1.7 & -27 & $8 \mathrm{H}-2,80-90$ & 65.85 & $\begin{array}{l}942 B \\
\text { Ship }\end{array}$ & 0.40 & 26.2 & -22.09 \\
\hline $9 \mathrm{H}-1,71-75$ & 74.81 & & 1 & 2.1 & -27.03 & $8 \mathrm{H}-4,63-65$ & 65.93 & & 0.25 & 31.3 & -23.13 \\
\hline $9 \mathrm{H}-2,19-20$ & 75.61 & Ship & 1.1 & 1.6 & -26.95 & $8 \mathrm{H}-2,120-125$ & 66.08 & $942 B$ & 0.34 & 12.5 & -23.09 \\
\hline & & & & & & & & & & & \\
\hline & 77.54 & & 1 & 2.4 & -27.04 & H-5, 35-37 & 67.15 & & 0.68 & 0.6 & -27.47 \\
\hline $9 \mathrm{H}-3,86-87$ & 77.69 & Ship & 1 & 2.2 & -26.86 & $8 \mathrm{H}-5,55-56$ & 67.35 & Ship & 0.49 & 1.0 & -26.13 \\
\hline $9 \mathrm{H}-5,71-75$ & 79.34 & & 1 & 2 & -27.17 & $8 \mathrm{H}-5,84-86$ & 67.64 & & 0.83 & 0.7 & -27.00 \\
\hline $9 \mathrm{H}-6,71-75$ & 80.84 & & 1.1 & 1.6 & -27.11 & $8 \mathrm{H}-5,122-124$ & 68.02 & & 0.81 & 1.0 & -26.89 \\
\hline $9 \mathrm{H}-7,31-32$ & 81.94 & Ship & 0.9 & 2.5 & -26.95 & $8 \mathrm{H}-6,25-27$ & 68.55 & & 0.86 & 0.2 & -26.75 \\
\hline $9 \mathrm{H}-7,71-75$ & 82.34 & & 0.9 & 1.7 & -27.06 & $8 \mathrm{H}-6,85-87$ & 69.15 & & 0.85 & 0.2 & -26.52 \\
\hline $10 \mathrm{X}-1,65-70$ & 84.25 & & 1 & 1.8 & -26.95 & $8 \mathrm{H}-6,119-120$ & 69.49 & Ship & 0.69 & 2.1 & -26.02 \\
\hline $10 \mathrm{X}-2,19-20$ & 85.29 & Ship & 0.8 & 3.3 & -26.99 & $8 \mathrm{H}-6,130-132$ & 69.60 & & 0.87 & 0.4 & -26.49 \\
\hline $10 X-3,65-70$ & 86.42 & & 0.7 & 1.2 & -26.74 & $8 \mathrm{H}-7,13-15$ & 69.93 & & 0.79 & 0.2 & -26.63 \\
\hline $10 X-4,15-16$ & 87.42 & Ship & 0.9 & 1.7 & -27.15 & $9 \mathrm{H}-1,25-28$ & 70.55 & & 0.89 & 0.6 & -26.74 \\
\hline $10 X-4,65-70$ & 87.92 & & 1 & 1.5 & -27.13 & $9 \mathrm{H}-1,75-78$ & 71.05 & & 0.87 & 1.5 & -26.61 \\
\hline $10 X-5,65-70$ & 89.42 & & 1 & 1.9 & -27.13 & $9 \mathrm{H}-1,126-128$ & 71.56 & & 0.85 & 0.9 & -26.60 \\
\hline $10 X-6,65-70$ & 90.69 & & 1 & 1.7 & -27.17 & $9 \mathrm{H}-2,25-28$ & 72.05 & & 0.80 & 1.2 & -26.59 \\
\hline $11 X-1,72-77$ & 93.82 & & 0.8 & 1.7 & -26.97 & $9 \mathrm{H}-2,77-79$ & 72.57 & & 0.80 & 1.6 & -26.55 \\
\hline $11 X-2,72-77$ & 95.32 & & 1 & 1.3 & -26.98 & $9 \mathrm{H}-4,23-25$ & 72.90 & & 0.78 & 2.2 & -26.39 \\
\hline $11 X-3,72-77$ & 96.82 & & 1.1 & 2.2 & -27.12 & $\mathrm{H}-4,75-77$ & 73.42 & & 0.90 & 2.1 & -26.65 \\
\hline $11 X-4,27-28$ & 97.87 & Ship & 1 & 1.7 & -26.89 & $9 \mathrm{H}-4,79-80$ & 73.46 & Ship & 0.71 & 16.1 & -25.97 \\
\hline $11 X-4,72-77$ & 98.32 & & 1 & 2.2 & -27.15 & $9 \mathrm{H}-4,126-128$ & 73.93 & & 0.91 & 2.4 & -26.71 \\
\hline $11 X-4,106-107$ & 98.66 & Ship & 0.8 & 2.2 & -27.12 & $9 \mathrm{H}-5,25-28$ & 74.42 & & 0.82 & 2.7 & -26.24 \\
\hline $11 X-5,77-82$ & 99.82 & & 1 & 1.7 & -27.18 & $9 \mathrm{H}-5,75-77$ & 74.92 & & 0.87 & 1.9 & -26.58 \\
\hline $11 X-5,109-110$ & 100.19 & Ship & 0.9 & 3.3 & -26.99 & $9 \mathrm{H}-6,16-18$ & 75.25 & & 0.85 & 1.7 & -26.66 \\
\hline & 101.28 & & 1 & 2.2 & -27.11 & & 75.52 & & 0.89 & 3.0 & -26.64 \\
\hline $12 X-1,79-84$ & 103.49 & & 1 & 1.5 & -27.1 & $9 \mathrm{H}-7,12-13$ & 75.55 & Ship & 0.83 & 3.4 & -26.75 \\
\hline $12 X-2,80-85$ & 105.00 & & 1 & 1.7 & -27.07 & $9 \mathrm{H}-7,75-77$ & 76.18 & & 0.83 & 2.0 & -26.65 \\
\hline $12 X-3,83-88$ & 106.53 & & 1 & 2.1 & -27.21 & $9 \mathrm{H}-7,126-128$ & 76.69 & & 0.92 & 2.0 & -26.65 \\
\hline $12 X-4,79-84$ & 107.99 & & 1 & 2.1 & -27.04 & $10 \mathrm{H}-1,26-28$ & 80.04 & & 0.80 & 4.6 & -26.47 \\
\hline $12 X-4,85-86$ & 108.05 & Ship & 0.94 & 2 & -27.05 & $10 \mathrm{H}-1,71-73$ & 80.43 & & 0.88 & 1.6 & -26.68 \\
\hline $12 X-5,35-36$ & 109.05 & Ship & 1.02 & 2 & -26.8 & $10 \mathrm{H}-1,127-129$ & 80.95 & & 0.84 & 2.4 & -26.62 \\
\hline & 109.52 & & 1.02 & 1.8 & -27.15 & $10 \mathrm{H}-2,26-28$ & 81.43 & & 0.90 & 1.7 & -26.67 \\
\hline $13 X-1,64-69$ & 113.04 & & 0.98 & 2.1 & -27 & $10 \mathrm{H}-2,73-75$ & 81.86 & & 0.73 & 6.1 & -26.34 \\
\hline $13 X-2,66-71$ & 114.56 & & 0.95 & 1.8 & -27.06 & $10 \mathrm{H}-2,124-125$ & 82.23 & & 0.86 & 1.2 & -26.67 \\
\hline $13 X-2,91-92$ & 114.81 & Ship & 0.96 & 2 & -26.9 & $10 \mathrm{H}-2,119-121$ & 82.29 & Ship & 0.57 & 14.2 & -26.47 \\
\hline & 116.09 & & 0.85 & 2.2 & -26.8 & $10 \mathrm{H}-3,26-28$ & 82.81 & & 0.76 & 2.7 & -26.58 \\
\hline $13 X-4,63-68$ & 117.51 & & 0.77 & 2.2 & -26.81 & $10 \mathrm{H}-3,74-76$ & 83.26 & & 0.74 & 2.8 & -26.61 \\
\hline $13 X-5,48-49$ & 118.88 & Ship & 0.75 & 1.8 & -26.69 & $10 \mathrm{H}-3,126-128$ & 83.74 & & 0.78 & 2.1 & -26.55 \\
\hline
\end{tabular}


Table 2 (continued).

\begin{tabular}{|c|c|c|c|c|c|c|c|c|c|c|}
\hline & & ole $938 \mathrm{~A}$ & & & & & & Iole 942B & & \\
\hline $\begin{array}{l}\text { Core, section, } \\
\text { interval }(\mathrm{cm})\end{array}$ & $\begin{array}{l}\text { Depth } \\
\text { (mbsf) }\end{array}$ & Remarks & TOC & $\mathrm{CaCO}_{3}$ & $\mathrm{~d}^{13} \mathrm{C}_{\text {org }}$ & $\begin{array}{l}\text { Core, section, } \\
\text { interval }(\mathrm{cm})\end{array}$ & $\begin{array}{l}\text { Depth } \\
\text { (mbsf) }\end{array}$ & Remarks & TOC & $\mathrm{CaCO}_{3}$ \\
\hline $13 X-5,63-68$ & 119.03 & & 0.8 & 1.7 & -26.79 & $10 \mathrm{H}-4,24-26$ & 84.18 & & 0.83 & 2.4 \\
\hline $13 \mathrm{X}-5,105-106$ & 119.45 & Ship & 0.85 & 2.1 & -26.65 & $10 \mathrm{H}-4,75-77$ & 84.65 & & 0.89 & 2.1 \\
\hline $14 \mathrm{X}-1,53-58$ & 122.53 & & 0.99 & 1.7 & -26.91 & $10 \mathrm{H}-4,126-128$ & 85.13 & & 0.74 & 3.2 \\
\hline $14 \mathrm{X}-1,127-128$ & 123.27 & Ship & 0.94 & 1.7 & -26.74 & $10 \mathrm{H}-5,26-28$ & $\begin{array}{l}85.59 \\
85.13\end{array}$ & & 0.88 & 1.7 \\
\hline $14 \mathrm{X}-2,53-58$ & 124.03 & & 0.92 & 0.9 & -26.42 & $10 \mathrm{H}-5,61-63$ & 85.91 & & 0.90 & 2.2 \\
\hline $14 \mathrm{X}-3,53-58$ & 125.53 & & 0.99 & 1.7 & -26.87 & $10 \mathrm{H}-5,66-67$ & 85.96 & Ship & 0.62 & 3.6 \\
\hline $14 \mathrm{X}-4,45-50$ & 126.95 & & 0.92 & 1.7 & -26.14 & $10 \mathrm{H}-5,126-128$ & 86.51 & & 0.74 & 3.7 \\
\hline $14 \mathrm{X}-4,67-68$ & 127.17 & Ship & 0.77 & 18.3 & -24.56 & $10 \mathrm{H}-6,26-28$ & 86.97 & & 0.89 & 1.7 \\
\hline $14 X-5,45-50$ & 128.45 & & 0.97 & 2 & -26.99 & $10 \mathrm{H}-6,76-78$ & 87.44 & & 0.78 & 2.8 \\
\hline $15 \mathrm{X}-1,39-40$ & 131.97 & Ship & 0.96 & 2.1 & -26.81 & $10 \mathrm{H}-6,126-128$ & 87.90 & & $\begin{array}{l}0.79 \\
0.79\end{array}$ & 1.8 \\
\hline $15 X-1,54-55$ & 132.12 & Ship & 0.92 & 2.2 & -26.83 & $10 \mathrm{H}-7,23-25$ & 88.33 & & 0.84 & $\begin{array}{l}1.0 \\
2.2\end{array}$ \\
\hline $15 X-1,75-80$ & 132.33 & & 0.95 & 1.7 & -26.67 & $10 \mathrm{H}-7,76-78$ & 88.82 & & 0.64 & 4.7 \\
\hline $15 \mathrm{X}-2,30-31$ & 133.40 & Ship & 0.74 & 6.2 & -23.71 & $11 \mathrm{H}-1,14-17$ & 89.44 & & 0.83 & 4.0 \\
\hline $15 X-2,75-80$ & 133.85 & & 0.88 & 0.8 & -26.59 & $11 \mathrm{H}-1,52-55$ & 89.76 & & 0.85 & 2.7 \\
\hline $15 X-3,75-80$ & 135.21 & & 0.83 & 3.8 & -26.33 & $11 \mathrm{H}-2,16-19$ & 90.15 & & 0.91 & 2.4 \\
\hline $15 \mathrm{X}-4,75-80$ & 136.71 & & 0.86 & $\begin{array}{l}3.0 \\
1.8\end{array}$ & -26.22 & $11 \mathrm{H}-2,52-55$ & 90.51 & & 0.78 & 3.6 \\
\hline $15 X-5,75-80$ & 138.21 & & 0.87 & $\begin{array}{l}1.0 \\
1.7\end{array}$ & -26.32 & $11 \mathrm{H}-2,111-114$ & 90.97 & & 0.86 & 3.0 \\
\hline $16 \mathrm{X}-1,75-80$ & 141.95 & & 0.63 & 0.8 & -25.6 & $11 \mathrm{H}-3,25-28$ & 91.74 & & 0.90 & 1.7 \\
\hline $16 \mathrm{X}-2,3-4$ & 142.73 & Ship & 0.66 & 6.8 & -23.33 & $11 \mathrm{H}-3,112-115$ & 92.12 & & 0.86 & 1.7 \\
\hline $16 \mathrm{X}-2,75-80$ & 143.45 & & 0.64 & 1.1 & -27.07 & $11 \mathrm{H}-4,10-13$ & 92.87 & & 0.92 & 2.5 \\
\hline $16 X-3,75-80$ & 144.95 & & 0.84 & 1.2 & -27.19 & $11 \mathrm{H}-4,52-55$ & 93.20 & & 0.92 & 2.5 \\
\hline $16 \mathrm{X}-4,83-88$ & 146.53 & & 0.88 & 2 & -27.22 & $11 \mathrm{H}-4,107-110$ & 93.75 & & 1.00 & 2.2 \\
\hline $16 \mathrm{X}-5,54-55$ & 147.74 & Ship & 0.86 & 1.5 & -26.95 & $11 \mathrm{H}-5,4-5$ & 93.97 & Ship & 0.80 & 4.5 \\
\hline $16 X-5,70-75$ & 147.90 & & 0.84 & 2.7 & -27.18 & $11 \mathrm{H}-5,52-55$ & 94.45 & 'ит & $\begin{array}{l}0.01 \\
1.01\end{array}$ & 2.1 \\
\hline $17 \mathrm{X}-1,74-79$ & 151.54 & & 0.87 & 2.2 & -27 & $11 \mathrm{H}-5,107-110$ & 95.00 & & 0.91 & 2.7 \\
\hline $17 \mathrm{X}-2,32-33$ & 152.62 & Ship & 0.91 & 4.2 & -26.44 & $11 \mathrm{H}-6,25-28$ & 95.68 & & 1.00 & 2.2 \\
\hline $17 \mathrm{X}-2,75-80$ & 153.05 & & 0.87 & 1.3 & -27.06 & $11 \mathrm{H}-6,52-55$ & 95.95 & & 1.01 & 2.9 \\
\hline $17 \mathrm{X}-3,75-80$ & 154.55 & & 0.94 & 2.3 & $\begin{array}{l}-26.81 \\
-261\end{array}$ & $11 \mathrm{H}-6,107-110$ & 96.50 & & 0.90 & 2.2 \\
\hline $18 \mathrm{X}-1,25-29$ & 160.65 & & 1.04 & 2.3 & -27.59 & $12 \mathrm{H}-1,26-29$ & 99.05 & & 1.00 & 2.5 \\
\hline $18 \mathrm{X}-1,70-75$ & 161.10 & & 1.04 & 2.5 & -27.6 & $12 \mathrm{H}-1,76-79$ & 99.54 & & 1.02 & 2.6 \\
\hline $18 \mathrm{X}-2,26-30$ & 161.64 & & 1.01 & 2.9 & -27.76 & $12 \mathrm{H}-1,125-128$ & 100.02 & & 1.00 & 2.9 \\
\hline $18 \mathrm{X}-2,70-75$ & 162.08 & & 1.05 & 2.7 & -27.69 & $12 \mathrm{H}-2,26-29$ & 100.32 & & 0.93 & 3.6 \\
\hline $18 \mathrm{X}-2,110-111$ & 162.48 & Ship & 1.06 & 2.5 & -27.64 & $12 \mathrm{H}-2,76-79$ & 100.80 & & 1.00 & 2.2 \\
\hline $18 \mathrm{X}-2,127-132$ & 162.65 & Мाт & 1.05 & 2.8 & $\begin{array}{l}-27.69 \\
-27\end{array}$ & $12 \mathrm{H}-2,125-128$ & 101.28 & & 0.88 & 3.2 \\
\hline $18 \mathrm{X}-3,26-30$ & 163.14 & & 1.06 & 2.8 & -27.65 & $12 \mathrm{H}-3,26-29$ & 101.78 & & 0.93 & 2.5 \\
\hline $18 X-3,70-75$ & 163.58 & & 0.92 & 2.4 & -27.59 & $12 \mathrm{H}-3,51-52$ & 102.03 & Ship & 0.82 & 3.9 \\
\hline $18 \mathrm{X}-3,127-132$ & 164.15 & & 0.97 & 4.2 & -27.77 & $12 \mathrm{H}-3,125-128$ & 102.75 & & 0.93 & 2.4 \\
\hline $18 \mathrm{X}-4,26-30$ & 164.64 & & 0.98 & 4.2 & -27.65 & $12 \mathrm{H}-3,76-79$ & 102.76 & & 0.72 & 2.7 \\
\hline $18 X-4,70-75$ & 165.08 & & 1.04 & 2.8 & -27.69 & $12 \mathrm{H}-4,25-28$ & 103.24 & & 0.96 & 2.1 \\
\hline $18 \mathrm{X}-\mathrm{CC}, 25-29$ & 165.78 & & $\begin{array}{l}1.08 \\
1.08\end{array}$ & 3.6 & $\begin{array}{l}-27.72 \\
-27\end{array}$ & $12 \mathrm{H}-4,73-76$ & 103.71 & & 0.90 & 2.4 \\
\hline $18 \mathrm{X}-\mathrm{CC}, 64-69$ & 166.17 & & $\begin{array}{l}1.00 \\
1.02\end{array}$ & 2.6 & -27.54 & $13 \mathrm{X}-1,25-27$ & 104.25 & & 0.87 & 4.6 \\
\hline $19 X-1,112-113$ & 171.22 & Ship & 0.88 & 1.8 & -27.74 & $13 \mathrm{X}-1,76-78$ & 104.76 & & 0.87 & 3.1 \\
\hline $19 \mathrm{X}-2,94-99$ & 172.54 & & 1.12 & 2.8 & -27.75 & $13 \mathrm{X}-1,125-127$ & 105.25 & & 1.02 & 2.7 \\
\hline $20 \mathrm{X}-1,65-70$ & 180.45 & & 1.05 & 3.1 & -27.97 & $13 \mathrm{X}-2,26-28$ & 105.76 & & 0.99 & 2.2 \\
\hline $20 X-2,60-65$ & 181.90 & & 0.99 & 4.2 & -27.68 & $13 \mathrm{X}-2,76-78$ & 106.26 & & 0.90 & 2.5 \\
\hline $20 \mathrm{X}-2,125-126$ & 182.55 & Ship & 0.31 & 2 & $\begin{array}{l}-27.22 \\
-27\end{array}$ & $13 \mathrm{X}-2,126-128$ & 106.76 & & $\begin{array}{l}.90 \\
1.00\end{array}$ & 1.8 \\
\hline $20 X-3,62-67$ & 183.42 & & 0.96 & 3.2 & -27.61 & $13 \mathrm{X}-3,25-27$ & 107.25 & & 0.99 & $\begin{array}{l}1.0 \\
1.6\end{array}$ \\
\hline $20 X-4,26-31$ & 184.56 & & 0.97 & 3.9 & -27.77 & $13 X-3,58-59$ & 107.58 & Ship & 0.90 & 3.2 \\
\hline $20 \mathrm{X}-4,74-75$ & 185.04 & Ship & 1 & 2.2 & -27.53 & $13 X-3,76-78$ & 107.76 & & 1.02 & 2.2 \\
\hline$-5,66-71$ & 186.46 & & 1.04 & 3.2 & -27.64 & $13 \mathrm{X}-3,126-128$ & 108.26 & & 0.84 & 3.7 \\
\hline $22 \mathrm{X}-1,93-98$ & 197.43 & & 1 & 3 & -27 & $13 \mathrm{X}-4,25-27$ & 108.75 & & 0.8 & 4.7 \\
\hline $22 \mathrm{X}-2,87-93$ & 198.87 & & 1 & 3.5 & -27.56 & $14 \mathrm{X}-1,26-28$ & 110.36 & & 1.0 & 2.7 \\
\hline$-3,77-82$ & 200.27 & & 1.01 & 2.8 & -27.6 & $14 \mathrm{X}-1,76-78$ & 110.86 & & 1.0 & 2.3 \\
\hline $4,30-31$ & 201.30 & Ship & 0.44 & 2.7 & -27.28 & $14 \mathrm{X}-1,126-128$ & 111.36 & & 0.99 & 1.6 \\
\hline $\begin{array}{l}4,54-69 \\
-4,64\end{array}$ & 201.64 & SIITP & 1 & 3.2 & $\begin{array}{l}-27.54 \\
-27\end{array}$ & $14 \mathrm{X}-2,26-28$ & 111.86 & & 0.85 & $\begin{array}{l}1.0 \\
4.7\end{array}$ \\
\hline $86-87$ & 201.86 & Ship & 0.97 & 2.2 & -27 & $14 \mathrm{X}-2,39-40$ & 111.99 & Ship & 0.94 & 2.0 \\
\hline $23 \mathrm{X}-1,75-8$ & 204.25 & & 0.93 & 2.7 & -27. & $14 \mathrm{X}-2,78-80$ & 112.38 & & 1.02 & 2.2 \\
\hline $23 \mathrm{X}-2,45-46$ & 205.45 & Ship & 1.01 & 2.2 & $\begin{array}{l}-27.41 \\
-2.45\end{array}$ & $14 \mathrm{X}-2,126-128$ & 112.86 & & $\begin{array}{l}1.02 \\
1.01\end{array}$ & 1.7 \\
\hline$-2,75-80$ & 205.75 & & 1.03 & 3 & -27.47 & $14 \mathrm{X}-3,26-28$ & 113.36 & & 0.9 & 3.6 \\
\hline $3,75-81$ & 206.99 & & 0.96 & 3.1 & -27.45 & $14 \mathrm{X}-3,82-84$ & 113.92 & & 1.02 & 2.2 \\
\hline $76-81$ & 213.86 & & 0.93 & 3.2 & -27 & $14 \mathrm{X}-3,126-128$ & 114.36 & & 1. & 2.2 \\
\hline & 215.36 & & 0.85 & 3.6 & -27 & $15 \mathrm{X}-1,76-79$ & 120.46 & & 1.0 & 2.3 \\
\hline$X-3,76-81$ & 216.86 & & 0.76 & 5.4 & -27. & $15 \mathrm{X}-1,126-129$ & 120.96 & & 1.10 & 1.3 \\
\hline$-4,59-60$ & $\begin{array}{l}210.19 \\
218.19\end{array}$ & Ship & 1 & 2.6 & -27. & $15 X-2,26-29$ & 121.46 & & 1.05 & 1.7 \\
\hline & 218.36 & & 1.05 & 3.6 & -27.4 & & 121.96 & & 1.0 & 2.0 \\
\hline $76-81$ & 219.86 & & 1.02 & 2.7 & -27.39 & $15 \mathrm{X}-2,126-129$ & 122.46 & & 1.06 & 2.1 \\
\hline $24-30$ & 223.04 & & 0.96 & 3.3 & -27 & $3,26-29$ & 122.96 & & 1.0 & 1.9 \\
\hline$X-2,72-78$ & 224.09 & & 1.01 & 3 & -27. & $15 X-3,47-48$ & 123.17 & Ship & 0.91 & 2.6 \\
\hline $25 \mathrm{X}-2,104-105$ & 224.41 & Ship & 0.93 & 2.2 & -272 & $15 X-3,76-79$ & 123.46 & & 1.02 & 2.0 \\
\hline $25 \mathrm{X}-\mathrm{CC}, 24-30$ & 225.11 & & 1.05 & 3.2 & -27.37 & $15 X-3,126-129$ & 123.96 & & $\begin{array}{l}1.02 \\
1.04\end{array}$ & 2.3 \\
\hline$-1,128-134$ & 233.58 & & 0.99 & 2.8 & -27.33 & $15 X-4,26-29$ & 124.46 & & 1.07 & 2.1 \\
\hline & & Ship & & 2.9 & & & & & 1. & 2.4 \\
\hline & 234.17 & & 1.05 & 2.6 & -27 & $15 \mathrm{X}-4,126-129$ & 125.42 & & & 2.1 \\
\hline $1,73-77$ & 242.53 & & 0.8 & 3.1 & -27 . & $15 X-5,26-28$ & 125.96 & & 1.08 & 2.4 \\
\hline$X-2,73-77$ & 244.03 & & 0.8 & 3.2 & -27.06 & $16 \mathrm{X}-1,26-28$ & 129.56 & & 0.98 & 1.7 \\
\hline X-3, 75-80 & 245.55 & & $\begin{array}{l}0.8 \\
0.8\end{array}$ & 3.9 & $\begin{array}{l}-27.11 \\
-2.11\end{array}$ & $\begin{array}{l}\mathrm{A}-1,20-20 \\
\mathrm{X}-2,86-89\end{array}$ & 131.66 & & $\begin{array}{l}.90 \\
1.00\end{array}$ & $\begin{array}{l}1.1 \\
2.6\end{array}$ \\
\hline$-4,45-47$ & 246.75 & Ship & 0.9 & 2 & -27.0 & $-2,126-128$ & 132.06 & & 1.0 & 1.7 \\
\hline & & & 0.9 & 2.7 & & & & & & \\
\hline & & & 0.9 & 2.7 & & & 133.16 & & 1.07 & 1.5 \\
\hline $27 \mathrm{X}-6,49-50$ & 249.79 & Ship & 0.8 & 1.7 & -27.05 & $16 \mathrm{X}-3,129-131$ & 133.59 & & 1.01 & 2.4 \\
\hline & 250.07 & & 0.9 & 2.3 & -27.2 & $16 \mathrm{X}-4,26-28$ & 134.06 & & 1.06 & 2.3 \\
\hline $28 \mathrm{X}-1,71-76$ & 252.11 & & 0.9 & 2.7 & -27.1 & $16 \mathrm{X}-4,76-77$ & 134.56 & Ship & 0.92 & 2.9 \\
\hline & 252.65 & & 0.9 & 3.2 & -27.27 & & 134.78 & & 1.0 & 2.3 \\
\hline & & & 0.9 & 3.2 & -27 & & & & 1. & 4.1 \\
\hline $28 \mathrm{X}-2,125-130$ & 254.15 & & 0.9 & 2.9 & -27.21 & $16 \mathrm{X}-5,26-28$ & 135.56 & & 1.08 & $\begin{array}{l}.1 \\
2.3\end{array}$ \\
\hline $28 \mathrm{X}-3,70-75$ & 255.10 & & 0.9 & 3.7 & -27.11 & $17 \mathrm{X}-1,25-28$ & 139.25 & & 1.01 & 2.5 \\
\hline
\end{tabular}


Table 2 (continued).

\begin{tabular}{|c|c|c|c|c|c|c|c|c|c|c|c|}
\hline \multicolumn{6}{|c|}{ Hole 938A } & \multicolumn{6}{|c|}{ Hole 942B } \\
\hline $\begin{array}{l}\text { Core, section, } \\
\text { interval }(\mathrm{cm})\end{array}$ & $\begin{array}{l}\text { Depth } \\
\text { (mbsf) }\end{array}$ & Remarks & TOC & $\mathrm{CaCO}_{3}$ & $\mathrm{~d}^{13} \mathrm{C}_{\text {org }}$ & $\begin{array}{l}\text { Core, section, } \\
\text { interval }(\mathrm{cm})\end{array}$ & $\begin{array}{l}\text { Depth } \\
\text { (mbsf) }\end{array}$ & Remarks & TOC & $\mathrm{CaCO}_{3}$ & $\mathrm{~d}^{13} \mathrm{C}_{\text {org }}$ \\
\hline $28 X-4,53-54$ & 255.43 & Ship & 0.9 & 3.5 & -27.21 & $17 X-1,75-78$ & 139.75 & & 1.08 & 2.2 & -27.48 \\
\hline $28 X-4,57-58$ & 255.47 & Ship & 0.6 & 2.8 & -27.19 & $17 \mathrm{X}-1,125-128$ & 140.25 & & 1.01 & 3.0 & -27.38 \\
\hline $28 \mathrm{X}-3,125-130$ & 255.65 & & 1 & 2.2 & -27.4 & $17 \mathrm{X}-2,15-18$ & 140.65 & & 1.01 & 2.1 & -27.32 \\
\hline $28 \mathrm{X}-4,80-85$ & 256.70 & & 0.9 & 3.5 & -27.18 & $17 \mathrm{X}-2,75-78$ & 141.22 & & 1.07 & 1.4 & -27.28 \\
\hline $28 \mathrm{X}-4,125-130$ & 257.15 & & 0.9 & 2.8 & -27.22 & $17 \mathrm{X}-2,125-128$ & 141.72 & & 1.07 & 2.3 & -27.49 \\
\hline $28 X-5,73-79$ & 258.13 & & 0.8 & 3.6 & -27.26 & $17 \mathrm{X}-3,25-28$ & 142.25 & & 1.02 & 1.8 & -27.20 \\
\hline $28 \mathrm{X}-5,124-130$ & 258.64 & & 0.8 & 3.2 & -27.22 & $17 X-3,75-78$ & 142.75 & & 1.00 & 2.1 & -27.33 \\
\hline $29 \mathrm{X}-1,73-77$ & 261.73 & & 0.9 & 3.9 & -27.23 & $17 \mathrm{X}-3,125-128$ & 143.25 & & 1.00 & 1.7 & -27.35 \\
\hline $29 X-2,73-77$ & 263.23 & & 0.8 & 3.4 & -27.25 & $17 \mathrm{X}-4,25-28$ & 143.75 & & 1.01 & 2.1 & -27.47 \\
\hline $29 X-3,77-81$ & 264.77 & & 0.8 & 3.3 & -27.28 & $17 \mathrm{X}-4,75-78$ & 144.25 & & 0.99 & 2.7 & -27.31 \\
\hline $29 X-3,114-115$ & 265.14 & Ship & 0.8 & 3.2 & -27.37 & $17 \mathrm{X}-4,79-80$ & 144.29 & Ship & 1.01 & 2.4 & -27.21 \\
\hline $29 X-4,68-72$ & 266.18 & & 0.9 & 3.2 & -27.24 & $17 \mathrm{X}-4,125-128$ & 144.75 & & 0.96 & 2.3 & -27.17 \\
\hline $29 X-5,68-72$ & 267.68 & & 0.9 & 3.5 & -27.28 & $17 X-5,14-17$ & 145.14 & & 0.96 & 2.8 & -27.23 \\
\hline $29 \mathrm{X}-6,50-51$ & 269.00 & Ship & 0.8 & 4.3 & -27.45 & $18 X-1,25-27$ & 148.85 & & 1.11 & 2.2 & -27.07 \\
\hline $30 \mathrm{X}-1,34-35$ & 270.94 & Ship & 0.9 & 2.2 & -27.25 & $18 X-1,75-77$ & 149.35 & & 1.08 & 2.8 & -27.18 \\
\hline $30 X-1,72-76$ & 271.32 & & 0.9 & 3.2 & -27.3 & $18 \mathrm{X}-1,125-127$ & 149.85 & & 1.12 & 2.2 & -27.22 \\
\hline $30 X-2,72-76$ & 272.82 & & 0.9 & 3.1 & -27.22 & $18 \mathrm{X}-2,25-27$ & 150.35 & & 1.11 & 2.7 & -27.13 \\
\hline $30 X-3,72-76$ & 274.32 & & 0.9 & 2.8 & -27.23 & $18 X-2,75-77$ & 150.85 & & 1.11 & 2.9 & -27.17 \\
\hline $30 X-3,88-89$ & 274.48 & Ship & 0.8 & 2.3 & -27.21 & $18 \mathrm{X}-2,125-127$ & 151.35 & & 1.09 & 2.5 & -27.06 \\
\hline $30 X-4,72-76$ & 275.82 & & 0.9 & 4.1 & -27.33 & $18 \mathrm{X}-3,25-27$ & 151.85 & & 1.03 & 2.2 & -27.13 \\
\hline $31 \mathrm{X}-1,57-62$ & 280.95 & & 0.9 & 3.4 & -27.19 & $18 X-3,75-77$ & 152.35 & & 1.10 & 2.6 & -27.17 \\
\hline $31 X-2,75-80$ & 282.45 & & 0.8 & 3.6 & -27.18 & $18 \mathrm{X}-3,125-127$ & 152.85 & & 1.08 & 3.1 & -27.08 \\
\hline $31 \mathrm{X}-2,102-103$ & 282.72 & Ship & 0.8 & 4.2 & -26.99 & $18 \mathrm{X}-4,25-27$ & 153.35 & & 1.09 & 2.9 & -27.09 \\
\hline $31 X-3,60-62$ & 283.80 & Ship & 0.9 & 2.2 & -27.32 & $18 X-4,75-77$ & 153.85 & Ship & 1.00 & 2.4 & -27.09 \\
\hline $31 X-3,75-80$ & 283.95 & & 0.8 & 3.7 & -27.3 & $18 \mathrm{X}-4,53-54$ & 153.89 & & 1.07 & 3.1 & -27.21 \\
\hline $32 X-1,75-80$ & 290.65 & & 0.9 & 4.4 & -27.1 & $18 \mathrm{X}-4,125-127$ & 154.35 & & 1.20 & 2.7 & -27.10 \\
\hline $32 X-2,75-80$ & 292.15 & & 0.8 & 4.1 & -27.17 & $18 X-5,25-27$ & 154.85 & & 1.05 & 2.3 & -27.08 \\
\hline $32 X-3,75-80$ & 293.65 & & 0.9 & 3.6 & -27.31 & $18 X-5,75-77$ & 155.35 & & 1.10 & 2.9 & -27.22 \\
\hline $32 X-4,75-80$ & 295.15 & & 0.9 & 3.9 & -27.08 & $18 X-5,125-127$ & 155.85 & & 1.09 & 3.6 & -27.24 \\
\hline $32 \mathrm{X}-4,93-94$ & 295.33 & Ship & 0.9 & 2.2 & -27.21 & $19 \mathrm{X}-1,25-27$ & 158.55 & & 1.08 & 3.2 & -27.29 \\
\hline $32 \mathrm{X}-5,27-28$ & 296.17 & Ship & 0.8 & 2.6 & -27.32 & $19 \mathrm{X}-1,72-74$ & 159.02 & & 1.09 & 3.1 & -27.24 \\
\hline $32 X-5,75-80$ & 296.65 & & 0.8 & 3.4 & -27.17 & $19 \mathrm{X}-1,125-127$ & 159.55 & & 1.05 & 3.4 & -27.16 \\
\hline $33 \mathrm{X}-1,71-76$ & 300.21 & & 0.8 & 3.2 & -27.2 & $19 \mathrm{X}-2,25-27$ & 160.05 & & 1.04 & 3.3 & -27.11 \\
\hline $33 \mathrm{X}-2,79-84$ & 301.79 & & 0.8 & 3 & -27.25 & $19 X-2,77-79$ & 160.57 & & 1.06 & 2.7 & -27.19 \\
\hline $33 X-2,146-147$ & 302.46 & Ship & 0.8 & 3.7 & -26.9 & $19 \mathrm{X}-2,125-127$ & 161.05 & & 1.05 & 3.2 & -27.11 \\
\hline $33 X-3,75-81$ & 303.25 & & 0.8 & 3.1 & -27.16 & $19 X-3,25-27$ & 161.55 & & 0.94 & 4.2 & -27.02 \\
\hline $33 \mathrm{X}-3,103-104$ & 303.53 & Ship & 0.8 & 2.9 & -27.22 & $19 X-4,36-37$ & 161.66 & & 1.07 & 2.3 & -27.29 \\
\hline \multirow[t]{15}{*}{$33 \mathrm{X}-4,77-82$} & 304.77 & & 0.7 & 3 & -27.21 & $19 X-3,75-77$ & 161.75 & & 1.03 & 1.8 & -27.14 \\
\hline & & & & & & $19 \mathrm{X}-3,125-127$ & 162.25 & & 1.03 & 2.2 & -27.25 \\
\hline & & & & & & $19 \mathrm{X}-4,25-27$ & 163.05 & Ship & 0.95 & 2.9 & -27.17 \\
\hline & & & & & & $19 X-4,75-77$ & 163.55 & & 1.08 & 2.2 & -27.19 \\
\hline & & & & & & $19 \mathrm{X}-4,125-127$ & 164.05 & & 1.09 & 2.1 & -27.17 \\
\hline & & & & & & $19 X-5,25-27$ & 164.55 & & 1.10 & 1.7 & -27.08 \\
\hline & & & & & & $19 X-5,77-79$ & 165.07 & & 1.10 & 2.2 & -27.25 \\
\hline & & & & & & $19 X-5,125-127$ & 165.55 & & 1.08 & 2.6 & -27.15 \\
\hline & & & & & & $20 \mathrm{X}-1,16-18$ & 168.06 & & 1.08 & 2.2 & -27.25 \\
\hline & & & & & & $20 \mathrm{X}-1,75-77$ & 168.65 & & 1.07 & 2.4 & -27.24 \\
\hline & & & & & & $20 \mathrm{X}-1,126-128$ & 169.16 & & 1.07 & 2.7 & -27.23 \\
\hline & & & & & & $20 \mathrm{X}-2,16-18$ & 169.56 & & 1.06 & 1.9 & -27.20 \\
\hline & & & & & & $20 \mathrm{X}-2,42-43$ & 169.82 & Ship & 1.01 & 1.9 & -27.12 \\
\hline & & & & & & $20 X-2,65-67$ & 170.05 & & 1.03 & 2.1 & -27.22 \\
\hline & & & & & & $20 \mathrm{X}-2,126-128$ & 170.66 & & 0.94 & 2.9 & -27.10 \\
\hline
\end{tabular}

Notes: Organic carbon isotope ratios are reported in per mil (\%o vs. PDB); "Ship" samples are shipboard carbonate samples measured for comparison. Corrected values from D. Piper, pers. comm., 1995.

\section{RESULTS AND DISCUSSION}

\section{Foraminifer Oxygen Isotope Records}

Oxygen isotope ratios provide stratigraphic information for all of the investigated sites (see Showers et al., this volume). At all holes from Sites 942, 938, and 940, foraminifers in sediments of Holocene age and from the warm interstadials of the penultimate interglacial are characterized by $\delta^{18} \mathrm{O}$ values between $1 \%$ o $-1.5 \%$ and $-2 \%$, whereas foraminifers contained in glacial sediments have higher $\delta^{18} \mathrm{O}$ values ranging between $-0.2 \%$ and $0.5 \%$ o. This allows a clear separation between sediment intervals representing maximum interglacial and glacial climate conditions, and respective periods of sea-level highstands and lowstands (Figs. 2-4).

In contrast to climatic extremes, the identification of depth intervals representing the periods of intermediate climate conditions and sea levels, as well as the fixing of stage boundaries or second-order isotopic events according to the global SPECMAP standard stack (Imbrie et al., 1984; Prell et al., 1986), was very difficult, if not impossible. Only for the upper $70 \mathrm{~m}$ of Hole 942A (Fig. 2), where the $\delta^{18} \mathrm{O}$ record is more similar to the standard stack, was identification of isotope stages possible for the last 135 k.y. (Showers et al., 1995).
During this period, the western part of the fan had been less affected by terrigenous input than the central fan, where the other two sites are located (Flood, Piper, Klaus, et al. 1995). Extremely high terrigenous sedimentation rates during glacials produced the 100- to 250-m-thick glacial levee and turbidite sequences that were recovered for isotope Stage 6 at Site 942 from the western fan, for Stages 2 to 4 at Site 938 from the upper central fan, and for Stage 2 at Site 940 retrieved from the most recent Amazon Channel complex. This pattern of very high glacial vs. low interglacial sedimentation rates can be observed also in the oxygen isotope records. They vary around typically glacial $\delta^{18} \mathrm{O}$ values over more than $100 \mathrm{~m}$ in the glacial core sections, whereas $\delta^{18} \mathrm{O}$ values representing the Holocene and the warm interstadials of isotope Stage 5 are restricted to very short depth intervals of a few meters or less (Figs. 2-4). The variability of $\delta^{18} \mathrm{O}$ values within the glacial sediment sections is predominantly attributed to millimeter- to centimeter-thick, silty or sandy turbidites intercalated in the hemipelagic muds that form the channel-levee sediments. Hence, biases in the isotope records due to reworked foraminifers are very likely. On the other hand, disturbances in the isotope records of planktonic foraminifers seem to be a function of the degree of distortion by the small-scale turbidites, which is indicated by the amount of millimeter-to centimeter-thick silty and sandy layers in the otherwise 


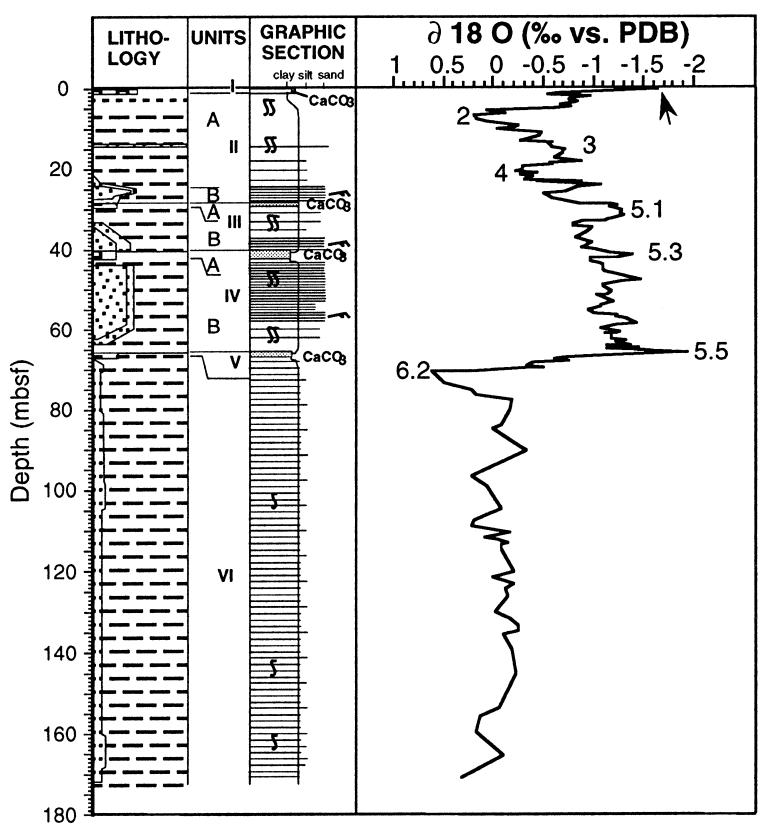

Figure 2. Graphic illustration of the macroscopic sediment description (adapted from Flood, Piper, Klaus, et al., 1995) and $\delta^{18} \mathrm{O}$ stratigraphy (from Showers et al., this volume) of Hole 942A. Black arrow indicates position of the respective topmost value.

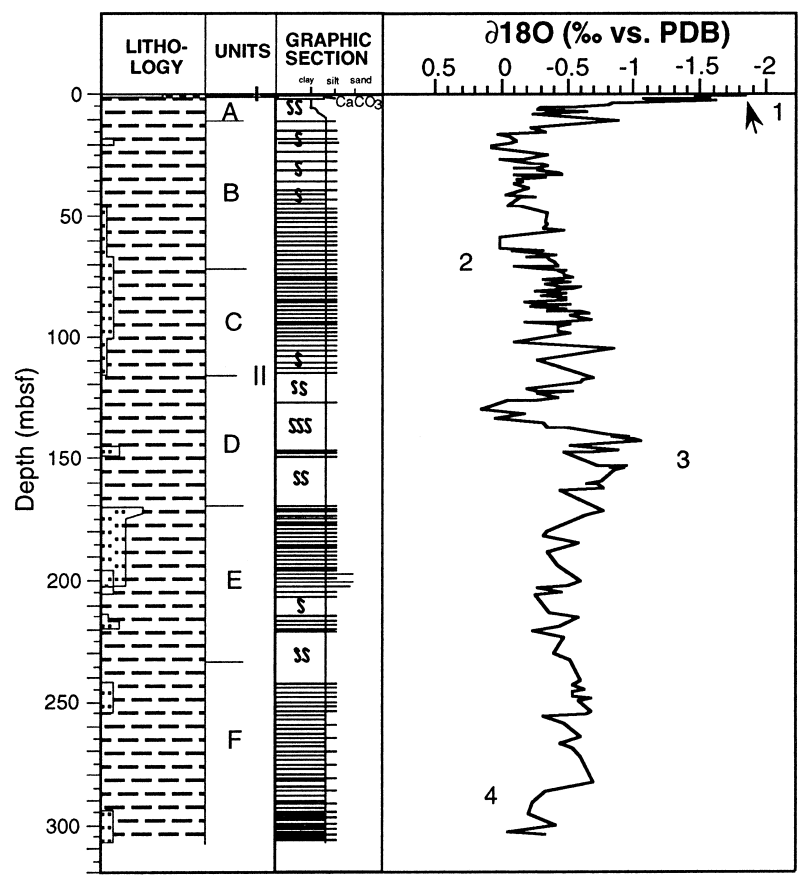

Figure 3. Graphic illustration of the macroscopic sediment description (adapted from Flood, Piper, Klaus, et al., 1995) and $\delta^{18} \mathrm{O}$ stratigraphy of Hole 938A. Black arrow indicates position of the respective topmost value.

hemipelagic bioturbated muds (see graphic sections in Figs. 2-4). We assume that at least in the bioturbated hemipelagic muds with low amounts of silt or sand, the stable isotope records roughly reflect changes in surface water conditions in the western equatorial Atlantic (see also discussion in Showers et al. and Maslin et al., this volume). As silt and sand layers increase in abundance and thickness, the reli-

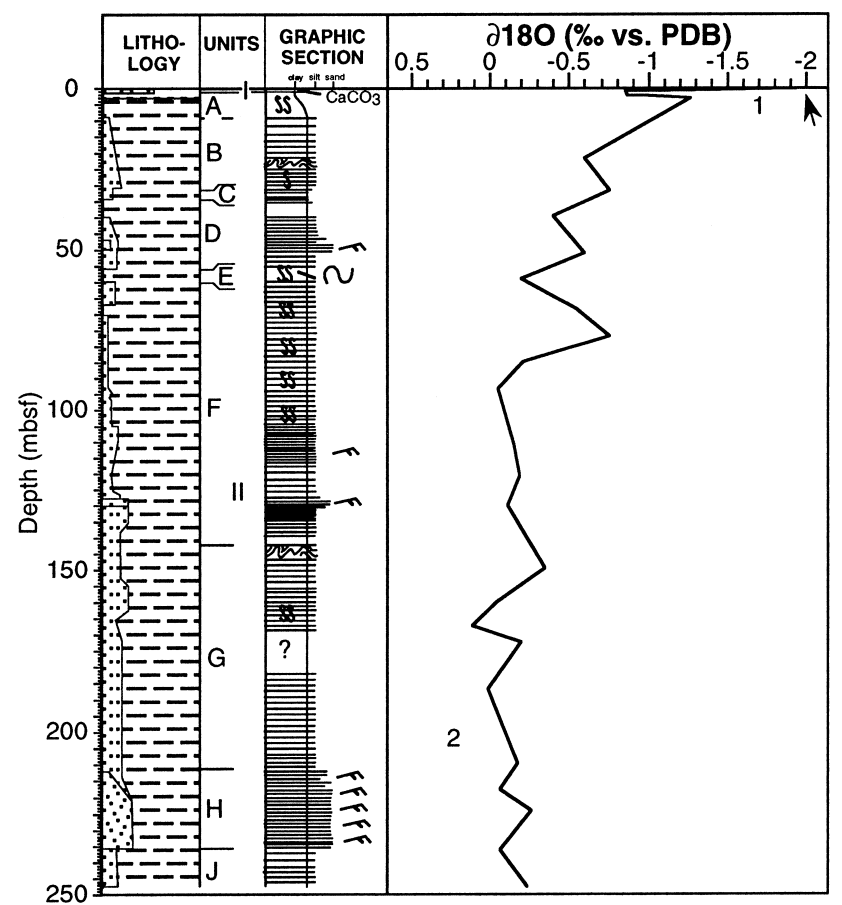

Figure 4. Graphic illustration of the macroscopic sediment description (adapted from Flood, Piper, Klaus, et al. 1995) and $\delta^{18} \mathrm{O}$ stratigraphy of Hole 940A. Black arrow indicates position of the respective topmost value.

ability of the stable isotope records decreases. For this reason, the best correlation of the $\delta^{18} \mathrm{O}$ record from Hole 942A with the SPECMAP stack is observed in the depth intervals of 0-25, 28-37, and 6375 mbsf (Fig. 2).

A strong glacial to Holocene decrease in $\delta^{18} \mathrm{O}$ values is documented in the upper $45 \mathrm{~m}$ of hemipelagic muds from Hole 938A (Fig. 3). At Site 940, the glacial sediments recovered had such low numbers of foraminifers that only a few core catcher samples provided enough tests of a distinct foraminifer species for stable isotope analysis at depth increments of $\sim 10 \mathrm{~m}$. However, a glacial to Holocene decrease similar to that at Site 938 is obvious as a climate signal in this 250$\mathrm{m}$-long $\delta^{18} \mathrm{O}$ record (Fig. 4). When compared with the $\delta^{18} \mathrm{O}$ record from Hole 942A, it follows that the bulk sedimentation rate for the LGM increased by a factor of almost 5 , respectively, from the westernmost part of the fan to the abandoned eastern levee complex at Site 938, and again from the latter site to Site 940 on the most recent Amazon Channel-levee Complex. Glacial sedimentation rates similar to those found at Sites 938 and 940 are observed in the western fan Site 942 only for Stage 6, which suggests an eastward propagation of the main active channel system over the last 140 k.y.

Aside from the glacial to Holocene $\delta^{18} \mathrm{O}$ decrease of $~ 2 \%$, and ignoring the high frequency change, the oxygen isotope record from Hole 938A reveals a smooth decrease from maximum glacial values at $20 \mathrm{mbsf}$ to intermediate values of about $-1 \%$ at $150 \mathrm{mbsf}$, increasing again to more glacial values of $\sim 0.3 \%$ o between $180 \mathrm{mbsf}$ and the bottom (Fig. 3). This pattern presumably represents the climate and sea-level change from the glacial Stage 4 (highest values at the bottom) through Stage 3 into the LGM. Lowest values $~ 150$ mbsf suggest a sediment age of $\sim 50$ ka corresponding to isotope Substage 3.3 in the SPECMAP stack. This age is corroborated by the shipboard magneto- and biostratigraphy, which delineate the geomagnetic Lake Mungo Excursion ( $32 \mathrm{ka})$ and the last occurrence of Pulleniatina obliquiloculata (>40 ka) at 145 and 149 mbsf, respectively (Flood, Piper, Klaus, et al., 1995). The magnetic age of $\sim 30 \mathrm{ka}$ and the oxygen isotope age of $\sim 50 \mathrm{ka}$ culminate within a relatively narrow depth 
interval of $5 \mathrm{~m}$. This suggests that intermediate climate and sea-level conditions during Stage 3 are represented by a very condensed sediment section compared to those of maximum glacial age during Stages 4 and 2. The more hemipelagic character of bioturbated muds between 120 and $170 \mathrm{mbsf}$, in contrast to the turbidite-rich sediments above and below this interval (Fig. 3, graphic section), supports this suggestion.

A pronounced excursion to higher $\delta^{18} \mathrm{O}$ values occurs in Hole 938A at $\sim 135 \mathrm{mbsf}$ (Fig. 3). Because this significant increase in $\delta^{18} \mathrm{O}$ values appears in the condensed hemipelagic sediment section, it is regarded as a real surface-water signal rather than a feature induced by reworked foraminifers. A similar excursion during Stage 3 in expanded glacial sediment sections has recently been found in planktonic $\delta^{18} \mathrm{O}$ records from the southwest African Margin (Jansen et al., 1996; Little et al., in press; Summerhayes et al., 1995). In these latter records, the $\delta^{18} \mathrm{O}$ maximum is rather poorly dated between 40 and 50 ka. Moreover, estimates of past sea-surface temperature (SST) in the eastern South Atlantic show a pronounced minimum at approximately the same age (Schneider et al., 1995a). In the eastern South Atlantic, this SST minimum, corresponding to the foraminifers' $\delta^{18} \mathrm{O}$ maximum in Stage 3, indicates an enhanced northward cold-water advection from the Southern Ocean with the Benguela Current (Jansen et al., 1996; Schneider et al., 1995a, 1996). A northward shift and intensification of the Benguela coastal upwelling system was also associated with this advectional event (Little et al., in press). According to the oxygen isotope chronology and the $P$. obliquiloculata datum, the $\delta^{18} \mathrm{O}$ excursion in Hole 938A correlates in age with the cold surfacewater event in the eastern South Atlantic during Stage 3. This excursion thus reveals strong evidence that cooling perturbations in the eastern South Atlantic are transferred to the western equatorial Atlantic by reduction of heat transport across the equator with the NBC. Furthermore, taking into account that during Stage 3 the global ice effect in the $\delta^{18} \mathrm{O}$ signal was less than in Stage 2, the strong excursion to maximum values at $135 \mathrm{mbsf}$ in Hole 938A may indicate that maximum cooling in the western Atlantic happened $\sim 40$ to $50 \mathrm{ka}$. and not during the LGM. However, this has to be regarded with caution, because we cannot exclude changes in the $\delta^{18} \mathrm{O}$ signal owing to freshwater induced salinity variations off the Amazon outfall, as previously described by Showers and Bevis (1988). Consequently, we need further information from other proxies that permit reconstruction of temporal changes in freshwater outflow from the Amazon (e.g., Schneider et al., 1995b).

\section{Foraminifer Carbon Isotope Records}

Attempts to explain variations in planktonic carbon isotope records are strongly hampered because of the poorly understood complexity of the genesis of the foraminifer $\delta^{13} \mathrm{C}$ signal (Hemleben et al., 1989; Schneider et al., 1994; Kroon and Darling, 1995). In paleoceanographic reconstructions, changes in the planktonic $\delta^{13} \mathrm{C}$ signal are variously attributed to local fluctuations in upwelling intensity and nutrient concentrations (Kroon and Ganssen, 1989; Sarnthein and Winn, 1990), to variations among atmospheric, terrestrial, and marine carbon reservoirs (Shackleton, 1977; Shackleton et al., 1992), to oceanwide fluctuations in surface and subsurface water $\mathrm{CO}_{2}$ concentrations (Berger and Vincent, 1986; Oppo and Fairbanks, 1989), or to changing atmosphere-ocean gas exchange rates in high latitudes (Charles and Fairbanks, 1990; Charles et al., 1993). In the case of the Amazon Fan $\delta^{13} \mathrm{C}$ records, this complexity is additionally complicated by repetitive and temporally variable turbidite-rich sediment sections. It is thus difficult to attribute the observed fluctuations in the Amazon records to anyone of the above paleoceanographic changes. Nevertheless, the highly expanded hemipelagic sediment intervals, which based on the $\delta^{18} \mathrm{O}$ records would seem to reflect a relatively undisturbed oceanographic signal, have the potential to give new insights into changes in the $\delta^{13} \mathrm{C}$ composition of dissolved surface seawater $\mathrm{CO}_{2}\left(\delta^{13} \mathrm{C}_{\mathrm{\Sigma CO} 2}\right)$ in the western Atlantic.

With the exception of the occasional extreme values, the Amazon Fan $\delta^{13} \mathrm{C}$ records of $G$. ruber at Sites 938 and 940 range between $0 \%$ and $1.5 \% \circ$ and are on average $\sim 1 \%$ o lower than those of $G$. sacculifer at Site 942 . Since the foraminifers analyzed were all from the same size fraction, this $1 \%$ o difference between both species may be explained by vital effects, especially different fractionation of calcite $\delta^{13} \mathrm{C}$ with respect to seawater $\delta^{13} \mathrm{C}_{\Sigma \mathrm{CO} 2}$ and different life habitat in the water column or by season (Kroon, 1988). At Site $942, \delta^{13} \mathrm{C}$ values of G. sacculifer vary between $1 \%$ and $2.5 \%$ over the last 125 k.y. (Fig. 5). Before Substage 5.5 (125 ka, $68 \mathrm{mbsf}$ in Hole 942A), the $G$. sacculifer $\delta^{13} \mathrm{C}$ record also shows a range of $\sim 1.5 \%$ o, but absolute values are shifted by $\sim 0.5 \%$ o to more negative ratios. Such a shift is similarly observed in a stacked planktonic $\delta^{13} \mathrm{C}$ record representing

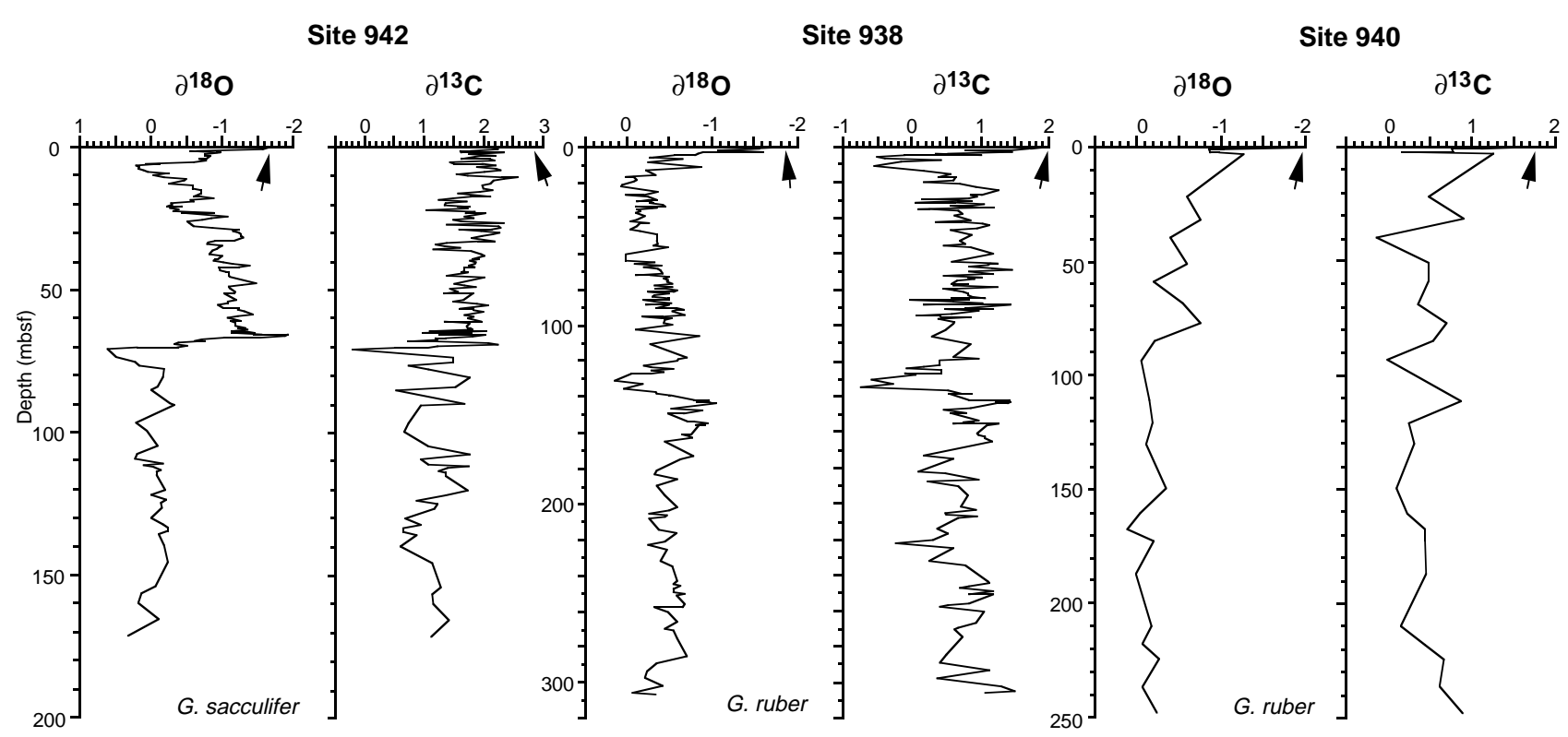

Figure 5. Foraminifer $\delta^{18} \mathrm{O}$ and $\delta^{13} \mathrm{C}$ records of G. sacculifer (Hole 942A), and G. ruber (Holes 938A and 940A). Black arrows indicate position of respective topmost value. 
changes in $\delta^{13} \mathrm{C}_{\Sigma \mathrm{CO} 2}$ over the last $260 \mathrm{k} . \mathrm{y}$. in the nutrient-depleted, western tropical Atlantic (Standard $\delta^{13} \mathrm{C}$ stack; Curry and Crowley, 1987). In this stacked record, $\delta^{13} \mathrm{C}$ values of $G$. sacculifer are $\sim 0.5 \%$ o more negative during late Stage $6(130-150 \mathrm{ka})$ compared with the average over the last 125 k.y., as observed in the record from Hole 942A. The excursions to more negative $\delta^{13} \mathrm{C}$ values at $33,18-22$, and 10 mbsf in Hole 942A (Fig. 5) are also present in the Curry and Crowley (1987) $\delta^{13} \mathrm{C}$ stack. The $\delta^{13} \mathrm{C}$ record from Site 942 is thus very similar to its equivalents from the open ocean. This suggests that surface water $\delta^{13} \mathrm{C}_{\mathrm{ECO} 2}$ changes over the western fan have not been very different from those in the larger western Atlantic during the upper Quaternary.

We cannot exclude that planktonic foraminifer records from off the Amazon estuary may have been affected by freshwater spikes in the $\delta^{13} \mathrm{C}$ signal, as was earlier described by Showers and Bevis (1988) for the Amazon and by Pastouret et al. (1978) for the Niger. In contrast to those studies, however, the negative $\delta^{13} \mathrm{C}$ excursions described here are not accompanied by shifts to lower $\delta^{18} \mathrm{O}$ values, which would be expected in case of a strong freshwater signal (Pastouret et al., 1978; Showers and Bevis, 1988). A greater detailed inspection of the foraminifer stable isotope records from the Amazon Fan, including denser sample intervals, may provide more conclusive evidence for freshwater signals in the stable isotope records from Leg 155 sites. Such an effort would also require a more detailed chronostratigraphy and the exclusion of isotopic spikes as a result of reworked foraminifers. Accelerator mass spectrometry (AMS) ${ }^{14} \mathrm{C}$ measurements from a short gravity core (GeoB 1513; Showers, unpubl. data) have shown age reversals in LGM sediments from the Amazon Fan that suggest reworking and redistribution of levee sediments on decimeter to meter scales. Nevertheless, from the data presented, we argue that observed changes in the planktonic $\delta^{13} \mathrm{C}$ record from Site 942 can be considered to approximate the general $\delta^{13} \mathrm{C}_{\Sigma \mathrm{CO} 2}$ signal of surface waters in the western Atlantic, which can itself be attributed to oceanwide reorganization of ocean circulation and/or to changes in $\delta^{13} C_{\Sigma \mathrm{CO} 2}$ composition (e.g., gas exchange and atmospheresurface ocean nutrient levels) in source regions of tropical Atlantic surface and subsurface waters (Curry and Crowley, 1987; Oppo and Fairbanks, 1989).

Two negative $\delta^{13} \mathrm{C}$ excursions, each $\sim 2 \%$ in amplitude can be observed in the hemipelagic sediment sections from Hole 938A at $\sim 5$ and $139 \mathrm{mbsf}$ (Fig. 5). A similar negative peak is observed in the $\delta^{13} \mathrm{C}$ record from Hole 933A for the last deglaciation (Maslin et al., 1995). The $2 \%$ o decreases in the Hole $938 \mathrm{~A}$ record are nearly twice the magnitude of corresponding peaks in pelagic planktonic $\delta^{13} \mathrm{C}$ records from the western Atlantic (Curry and Crowley, 1987). Because these negative $\delta^{13} \mathrm{C}$ peaks in the $G$. ruber record coincide with high glaciallike $\delta^{18} \mathrm{O}$ values, rather than with particularly low values, we exclude enhanced freshwater flux as the mechanism responsible for these excursions (see discussion above). Instead, the negative $\delta^{13} \mathrm{C}$ excursions may reflect changes in the Atlantic large-scale surface circulation, including the Benguela Current off Southwest Africa, the South Equatorial Current, and the NBC that flows along the Northeast Brazilian margin into the Caribbean Sea. Evidence for such a circulation signal originates from a few highly expanded planktonic $\delta^{13} \mathrm{C}$ records spanning the last $20 \mathrm{k} . \mathrm{y}$. The southernmost records, with a strong negative $\delta^{13} \mathrm{C}$ shift of $\sim 1 \%$, are known from the Antarctic polar front region and are dated with a radiocarbon AMS age of $13.8 \mathrm{ka}$ (Charles and Fairbanks, 1990). Farther north, a planktonic foraminifer record off Northern Namibia reveals a similar significant $\delta^{13} \mathrm{C}$ decrease with nearly the same age (13 ka, conventional ${ }^{14} \mathrm{C}$ method) and magnitude (Schneider et al., 1992). Following the surface circulation from the South Atlantic into the western North Atlantic, the $\delta^{13} \mathrm{C}$ excursion at $5 \mathrm{mbsf}$ in Hole 938A (roughly at $14 \mathrm{ka}$, according to the $\delta^{18} \mathrm{O}$ stratigraphy) is the next observation of this event. Finally, the same signal is observed in a new record from the Caribbean Sea (Peterson et al., 1995). All these records are characterized by much higher sedimentation rates than pelagic sediments, and because this southern ocean event had a short duration of 500 to $1000 \mathrm{yr}$ (Schneider et al., 1992), it is only clearly recorded by $\delta^{13} \mathrm{C}$ records from very expanded sediment sections. Together, these records point to a negative $\delta^{13} C_{\Sigma \mathrm{CO} 2}$ signal originating at high latitudes in the southern ocean and being transferred to the Northern Hemisphere via surface circulation.

The polar front region is a sensitive recorder of variations in southern ocean nutrient content and atmospheric-sea gas exchange. Both processes are presumably documented in the $\delta^{13} C_{\Sigma \mathrm{CO} 2}$ signal (Charles and Fairbanks, 1990). Polar surface waters set the preformed $\delta^{13} C_{\Sigma \mathrm{CO} 2}$ conditions for northward flowing subsurface waters, which from a simple point of view, feed the coastal and equatorial upwelling areas in the South Atlantic. Past nutrient or atmospheric $\mathrm{CO}_{2}$ perturbations in the southern ocean therefore may be reflected in $\delta^{13} \mathrm{C}_{\Sigma \mathrm{CO} 2}$ of the upwelled subsurface waters in South Atlantic upwelling areas and transported via surface currents into the North Atlantic. This model is in conflict, however, with another hypothesis that explains strong negative foraminifer stable isotope excursions during the last deglaciation as the result of excess meltwater flow to the Atlantic induced by melting of tropical and subtropical mountain glaciers (Showers and Margolis, 1985; Showers and Bevis, 1988). Both potential mechanisms need further evaluation with planktonic $\delta^{13} \mathrm{C}$ records retrieved from outside the Amazon freshwater plume. Additional information on the timing and extension of $\delta^{13} \mathrm{C}$ excursions is especially needed to enable correlation with other detailed records of changes in Atlantic surface water circulation; for example, the onset of the conveyor after the LGM (Broecker and Denton, 1989; Imbrie et al., 1993).

\section{Organic Carbon, Carbonate, and $\delta^{13} \mathrm{C}_{\text {org }}$ Records}

According to their stratigraphic units, the hemipelagic sediments from Holes 938A, 940A, and 942A reveal the opposing patterns in organic and inorganic carbon content, as well as in organic carbon isotope character (Figs. 6-8), that are well known from previous studies of sediment cores containing the LGM to Holocene period (Damuth and Kumar, 1975; Damuth et al., 1977; Showers and Angle, 1986). In general, sediments from intervals representing maximum warm climate and sea-level highstands that occurred during oxygen isotope Stage 1 and Substages 5.1, 5.3, and 5.5, are characterized by between 10 and $35 \mathrm{wt} \%$ calcium carbonate and 0.2 and $0.4 \mathrm{wt} \%$ TOC (Holes 938A and 940A: upper $0.5 \mathrm{~m}$; Hole 942A: upper $0.5 \mathrm{~m}$ and $66.5 \mathrm{mbsf}$ ). In sediments from maximum glacial periods with minimum sea level (isotope Stages 2, 4, and 6), TOC contents vary between 0.8 and $1 \mathrm{wt} \%$, and calcium carbonate concentrations are generally less than $3 \mathrm{wt} \%$ (Hole 938A: 1-307 mbsf; Hole 940A: 1-248 mbsf; Hole 942A: 1-4 mbsf, 23-7 mbsf, 67-172 mbsf). The $\delta^{13} \mathrm{C}_{\text {org }}$ values follow this inverse pattern of TOC and $\mathrm{CaCO}_{3}$ content for the upper Quaternary climate extremes. Maximum glacial TOC contents reveal isotope ratios of about $-27 \%$, whereas values ranging between $-22 \%$ and $-19 \%$ are characteristic of TOC-poor sediments from periods of maximum warmth and sea-level highstands. Periods of intermediate climate conditions and sea level, like during oxygen isotope Stage 3 and Substage 5.2 (6-20 mbsf and 30-40 mbsf, respectively, in Hole 942A) typically contain slightly more carbonate (5-10 wt\%) and less TOC $(0.5-0.7 \mathrm{wt} \%)$, with intermediate $\delta^{13} \mathrm{C}_{\text {org }}$ values varying about $-24 \%$. Also in the hemipelagic sediment sequence between 125 and 145 mbsf in Hole 938A, presumably representing oxygen isotope Stage 3 (compare Figs. 3, 7), there is a tendency for slightly enhanced carbonate contents coincident with reduced TOC concentrations and intermediate $\delta^{13} C_{\text {org }}$ values of about $-24 \%$. Sediment sections containing high amounts of millimeter- to centimeter-thick silt and sand layers or single decimeter-thick turbidites, illustrated by the dense line pattern in the graphic sections of Figures $6-8$, are characterized by very low carbonate contents and low (0.05-0.4 wt\%) TOC contents with $\delta^{13} \mathrm{C}_{\text {org }}$ values of $-26 \%$ to $-28 \%$. This can be seen, for example, in Hole 942A between 44-63 mbsf, in Hole 938A between 170 and 200 mbsf, and in Hole 940A at 130 mbsf. 


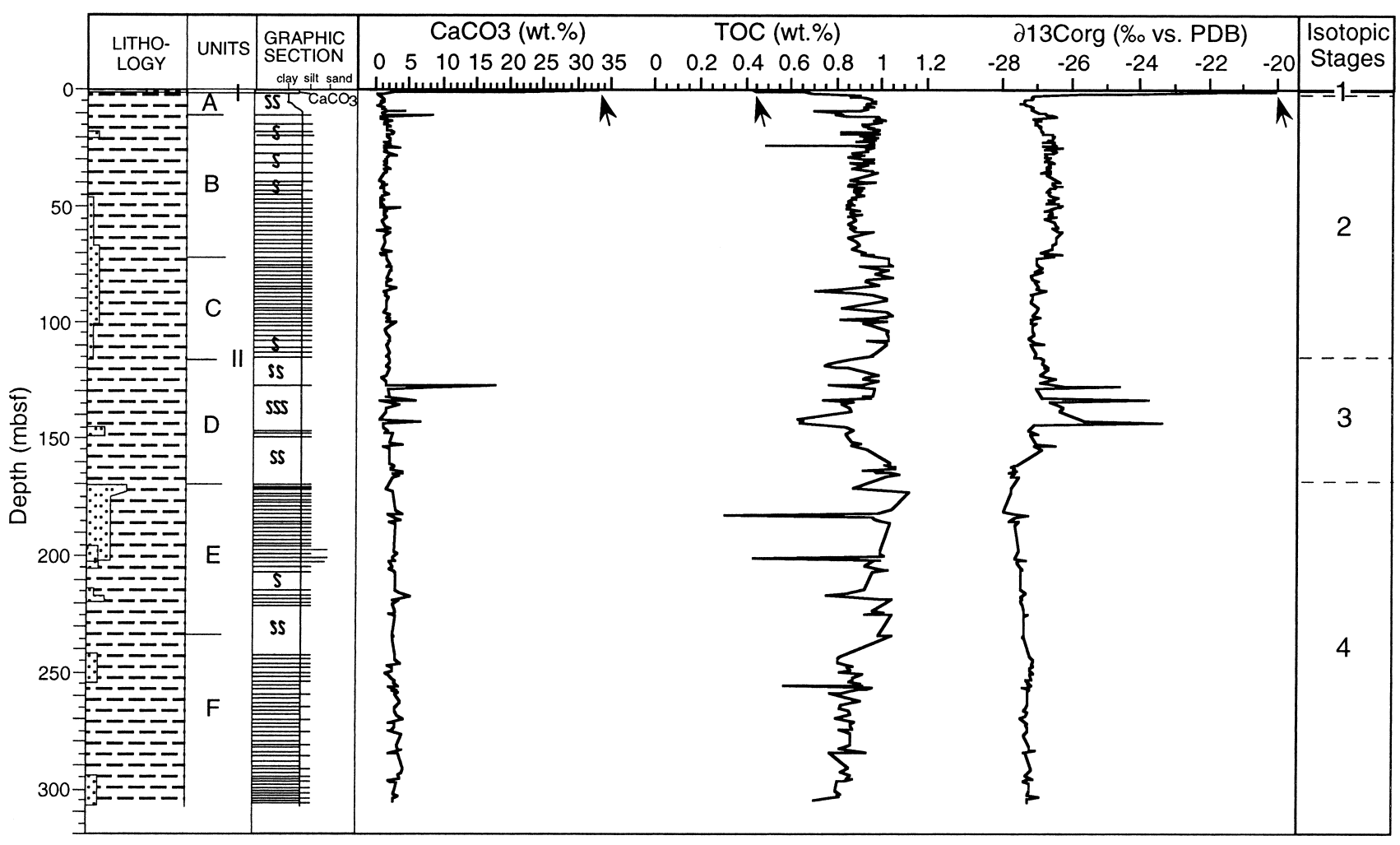

Figure 6. Graphic illustration of the macroscopic sediment description (adapted from Flood, Piper, Klaus, et al., 1995) records of $\mathrm{CaCO}_{3}$, TOC, and $\delta^{13} \mathrm{C}_{\text {org }}$, and isotopic stages for Hole 938A. Black arrows indicate position of respective topmost value.

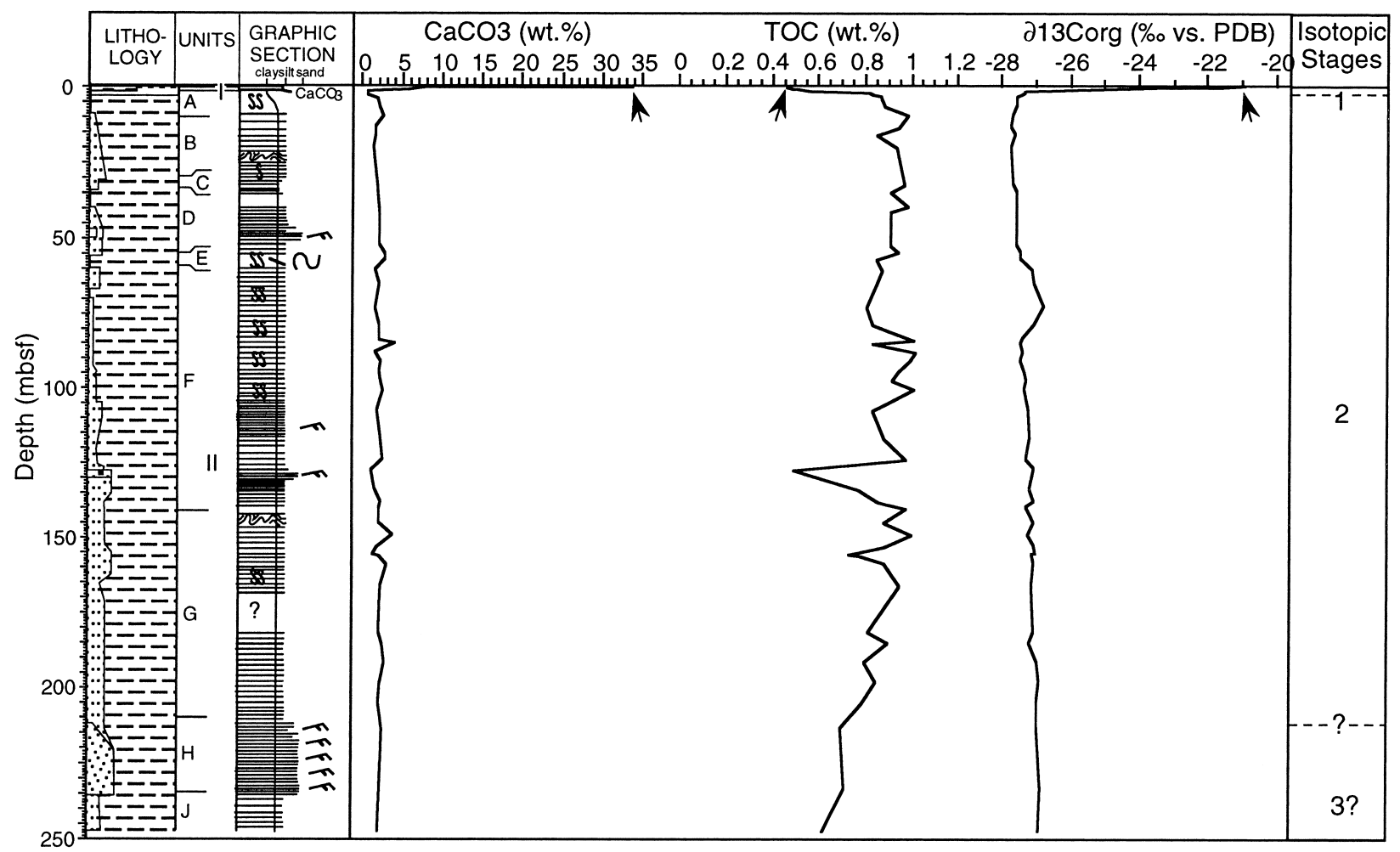

Figure 7. Graphic illustration of the macroscopic sediment description (adapted from Flood, Piper, Klaus, et al., 1995) records of $\mathrm{CaCO}_{3}$, $\mathrm{TOC}$, and $\delta^{13} \mathrm{C}_{\text {org }}$, and isotopic stages for Hole 940A. Black arrows indicate position of respective topmost value. 


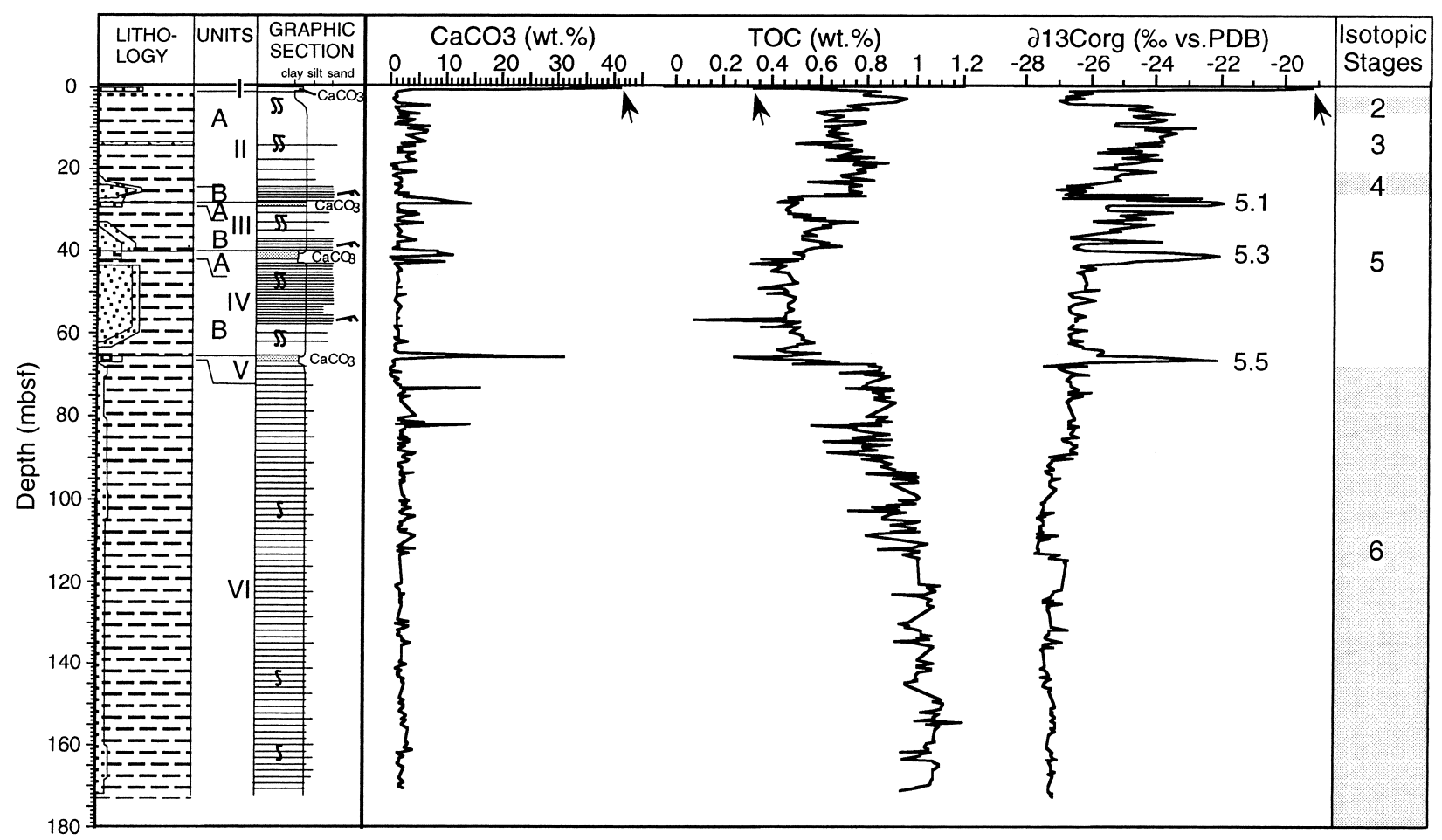

Figure 8. Graphic illustration of the macroscopic sediment description (adapted from Flood, Piper, Klaus, et al., 1995) records of $\mathrm{CaCO}_{3}$, TOC, and $\delta^{13} \mathrm{C}_{\text {org }}$, and isotopic stages for Hole 942A. Black arrows indicate position of respective topmost value.

Most of the variations observed in the records of $\mathrm{TOC}, \mathrm{CaCO}_{3}$, and $\delta^{13} \mathrm{C}_{\text {org }}$ can be explained simply by shifts from pelagic carbonaterich sedimentation at rates similar to open ocean sites to terrigenous alluvial fan deposition at very high sedimentation rates $(5$ to $20 \mathrm{~m} /$ k.y.) and vice versa. Periods of predominant contribution by marine constituents to the Amazon Fan, in other words times of minimum terrigenous dilution, are characterized by highest $\mathrm{CaCO}_{3}$ and $\delta^{13} \mathrm{C}_{\text {org }}$ values, but lowest TOC concentrations. Carbonate is derived predominantly from coccolithophorids and foraminifers (Maslin and Mikkelsen, this volume), and the $\delta^{13} \mathrm{C}_{\text {org }}$ values are typical for organic matter produced by marine phytoplankton in the tropical ocean (Westerhausen et al., 1993; Müller et al., 1993). In Holes 938A and 940A, the pelagic type of sediment is found only in the Holocene sections (Figs. 6, 7), as was the case for short gravity or piston cores investigated before. The period of highest sea level during the penultimate interglacial is documented in Hole $942 \mathrm{~A}$ at $\sim 66.5 \mathrm{mbsf}$. The $\mathrm{CaCO}_{3}$ contents and $\delta^{13} \mathrm{C}_{\text {org }}$ values at that depth in Hole $942 \mathrm{~A}$ do not reach Holocene values, most likely because the exact core depth for the penultimate climatic optimum was missed owing to the wide sampling intervals. The other two sea-level maxima corresponding to Substages 5.3 and 5.1, which were probably on the order of $30 \mathrm{~m}$ lower than at Substage 5.5 (see SPECMAP stack as an indicator for relative changes in sea level; Imbrie et al., 1984), are indicated by shifts to a sediment character more similar to an interglacial one. The records from Holes 938A, 940A, and 942A are thus consistent with the Amazon Fan sedimentation model, that during periods of highest sea level most of the terrigenous Amazon River load is deposited on the shelf or transported westward by alongshore currents and does not reach the fan (Damuth and Kumar, 1975; Damuth, 1977). In contrast, as can be seen from the Hole 942A record (Fig. 8), a drop in sea level from oxygen isotope Substages 5.5 to 5.4 of only $30-50 \mathrm{~m}$ (from SPECMAP-based estimates) resulted in erosion of shelf sediments and a predominance of sandy terrigenous sedimentation over the western fan. Showers and Bevis (1988) similarly estimated that a sea level of $\sim 40 \mathrm{~m}$ below the modern highstand was the threshold level for cessation of dominantly terrigenous sedimentation over the central fan during the transition from the LGM to the Holocene.

It was suggested earlier by Milliman et al. (1975) that, during periods of glacial sea-level lowstand, Amazon sediment dispersal was guided by shelf-cutting canyons directly to the upper fan (Fig. 9). In glacial sections from Holes 938A, 940A, and 942A, the sedimentary terrigenous end-member is defined by the lowest $\delta^{13} \mathrm{C}_{\text {org }}$ and $\mathrm{CaCO}_{3}$ values, and the highest TOC contents. Under modern conditions, $\delta^{13} \mathrm{C}_{\text {org }}$ values of $-27 \%$ to $-26 \%$ are characteristic of particulate organic carbon transported in the lower reaches of the Amazon River (Cai et al., 1988) and deposited in the Holocene mud wedge near the river mouth (Showers and Angle, 1986). Outer shelf sediments at the forward rim of the prograding Amazon mud wedge reveal heavier values of about $-24 \%$ o to $-22 \%$, owing to the mixing of terrigenous carbon with marine organic matter. Further away from the Amazon outfall, at the shelf break outside the mud wedge, values of about $-22 \%$ o indicate the growing dominance of marine organic carbon in surface sediments (Showers and Angle, 1986). The continuously low $\delta^{13} \mathrm{C}_{\mathrm{org}}$ values of about $-27 \%$ in glacial sediment sections from the ODP holes (Figs. 6-8) thus corroborate the assumption that, during glacial sea-level lowstands, the terrigenous Amazon sediment load was directly transported through the distributary channel systems onto the fan. Otherwise, if the westward transport of Amazon sediments was also prevalent during glacial sea-level lowstands, we would expect proportionately more marine organic carbon that would lead to a shift towards heavier $\delta^{13} \mathrm{C}_{\text {org }}$ values as observed in outer shelf deposits today. This may have been the case during phases of intermediate sea level, for example, during oxygen isotope Stage 3. However, for maximum lowstands of sea level, most of the terrigenous Amazon sediment load was deposited over the fan. The glacial deposits comprise an estimated $99 \%$ of sediments retrieved from Holes 938A and 940A, indicating the vast amount of terrigenous material transported to the central fan during the isotope Stages 2 and 4. Similar high rates of terrestrial deposition occurred in the western fan area during the penultimate glacial (Stage 6). 


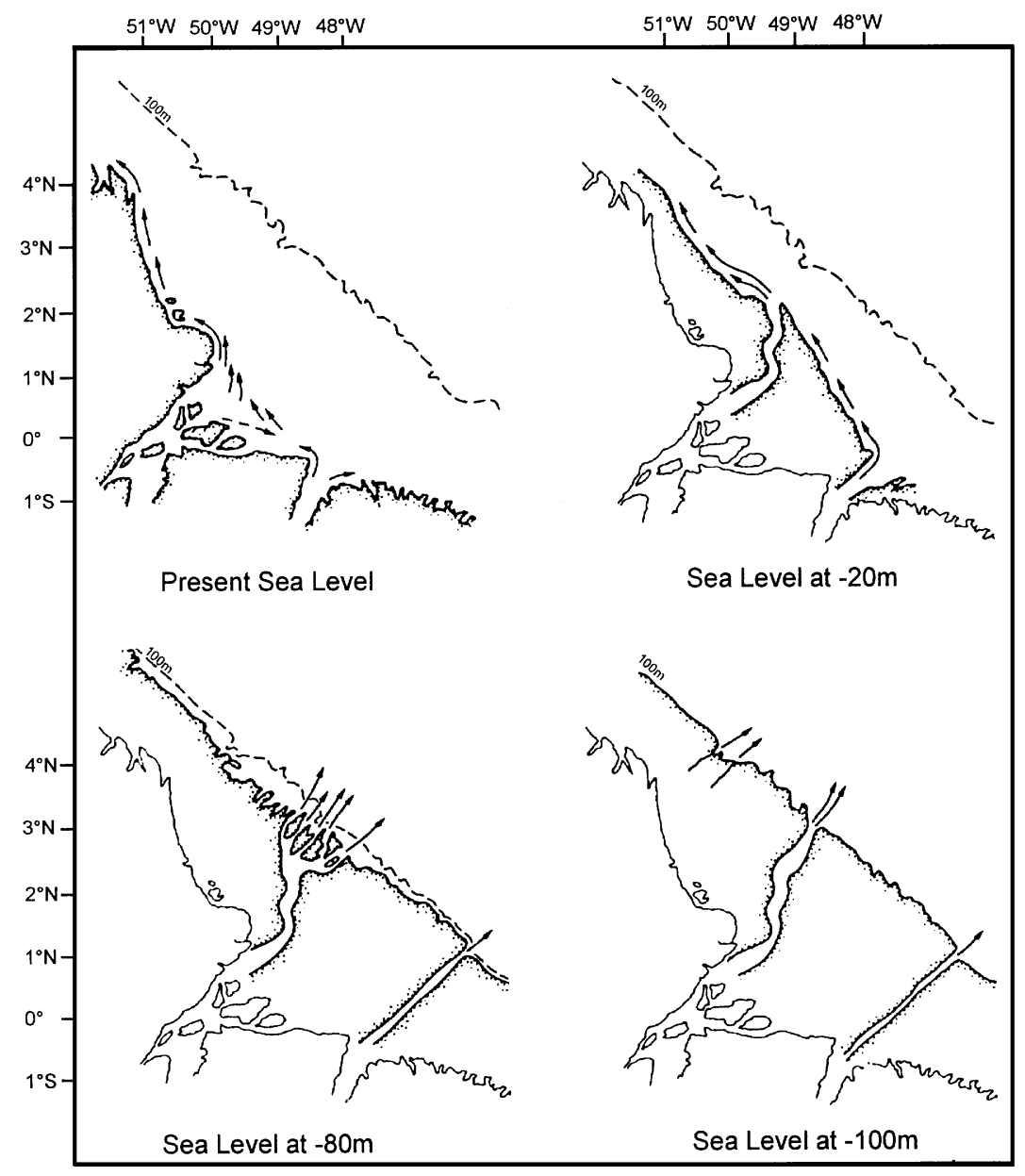

Figure 9. Model of Quaternary sedimentation off the Amazon River during high- and lowstands of sea level. Arrows delineate main routes of sediment dispersal. Dashed line refers to present $100 \mathrm{~m}$ isobath, which approximates the shelf edge (adapted from Milliman et al., 1975).
Noteworthy is the relative constant TOC content between 0.8 and $1 \mathrm{wt} \%$, in glacial terrigenous sediments. These values are similar to the TOC contents in surface sediments of the mud wedge on the shelf off the Amazon mouth. This organic matter seems to represent the average nonrefractory organic residue that survives deposition on the shelf and later transport to or direct deposition on the upper and middle fan. No indication exists from bulk $\delta^{13} \mathrm{C}_{\text {org }}$ values that this organic carbon residue, sampled preferentially from hemipelagic or turbiditefree terrigenous levee muds, may contain different plant source material that would point to climate-related changes in the vegetation in the Amazon catchment area (e.g., rainforest or grassland), as was suggested by Bird et al. (1991). Shifts to more positive $\delta^{13} \mathrm{C}_{\text {org }}$ values, observed in more condensed hemipelagic sediment intervals of isotope Substage 5.2 and Stage 3, coincide with reduced TOC and slightly enhanced $\mathrm{CaCO}_{3}$ contents. Hence, these increases in $\delta^{13} \mathrm{C}_{\text {org }}$ are attributed to a decrease of terrigenous dilution of the marine sediment component at times of intermediate climate and sea-level conditions. This would also explain the lower sedimentations rates during these climate stages (Flood, Piper, Klaus, et al., 1995: site summaries Holes 938A and 942A; also above discussion of oxygen isotopes for Stage 3 in Hole 938A; Fig. 2).

\section{CONCLUSIONS}

We have investigated sediment records from Leg 155, Sites 938, 940 , and 942 , in order to gain new insights to hydrographic changes in western Atlantic surface circulation and to test the model of Amazon Fan sedimentation patterns derived from previously investigated shorter gravity or piston cores. The results from analysis of TOC and $\mathrm{CaCO}_{3}$ concentrations, of $\delta^{13} \mathrm{C}_{\text {org }}$, as well as $\delta^{18} \mathrm{O}$ and $\delta^{13} \mathrm{C}$ values of the planktonic foraminifers G. ruber (pink) and G. sacculifer (see also Showers et al., this volume), lead to the following conclusions.

First, stable oxygen isotope records of planktonic foraminifers reveal an upper Quaternary pattern similar to those observed in isotope records from the open ocean and thus confirm the preliminary shipboard bio- and magneto-stratigraphic chronologies. Moreover, the isotopic excursions detected in the planktonic $\delta^{18} \mathrm{O}$ and $\delta^{13} \mathrm{C}$ records in relatively undisturbed sediment sections can be tentatively related to similar events observed in other records from the Antarctic, the southwest African Margin, and the Caribbean Sea. This suggests that attended changes in NBC hydrography off the Amazon are closely connected to fluctuations in oceanwide surface circulation. No clear evidence is found in support of a freshwater discharge signal in isotope records of planktonic foraminifers.

Second, variations in TOC and $\mathrm{CaCO}_{3}$ content, as well as $\delta^{13} \mathrm{C}_{\text {org }}$, provide clear evidence that the model of fan sedimentation previously suggested for the last 20 k.y. (Damuth and Kumar, 1975; Damuth, 1977) is also valid for the last 140 k.y. Fan deposition of marine biogenic matter vs. terrigenous input is predominantly controlled by upper Quaternary sea-level changes. During the Holocene and the penultimate interglacial maximum (Substage 5.5), corresponding with sea-level highstands, fan sedimentation was dominated by fluxes of calcite from coccoliths and foraminifers and of marine TOC with $\delta^{13} \mathrm{C}$ values of about $-20 \%$. During glacial and interglacial sea-level lowstands (Stages 2, 4, 6, and Substages 5.2 and 5.4), deposition of terrigenous material, including terrestrial organic carbon, occurred at very high rates $(\sim 5-20 \mathrm{~m} / \mathrm{k} . \mathrm{y}$.) along distributary channels. Low 
$\delta^{13} \mathrm{C}_{\text {org }}$ values of about $-27 \%$ in all glacial deposits indicate that during sea-level lowstands, the Amazon River load was discharged directly to the upper and middle fan in contrast to the modern situation, where the sediments are being redistributed westward with the NBC. Conditions intermediate to maximum and minimum sea level are represented by condensed sediment sections belonging to oxygen isotope Substage 5.2 and Stage 3. At these periods, terrigenous dilution of the pelagic sediment signal was not as high as during glacial maxima, which is indicated by organic and inorganic carbon contents and $\delta^{13} C_{\text {org }}$ ratios intermittent between the interglacial and glacial extremes.

\section{ACKNOWLEDGMENTS}

We thank the crew and scientific party of Leg 155 for a successful venture at sea and ODP for samples. In addition, we are indebted to H. Buschhoff, R. Kreutz, and B. Meyer, who took care of sample analysis at the Fachbereich Geowissenschaften, University of Bremen. We would like to thank F. Jansen, L. Peterson, and an anonymous reviewer for their suggestions which really improved the submitted manuscript. Editorial help from ODP staff and M. Little is also acknowledged. Financial support was provided by the Deutsche Forschungsgemeinschaft (We 992/18).

\section{REFERENCES}

Berger, W.H., and Vincent, E., 1986. Deep-sea carbonates: Reading the carbon-isotope signal. Geol. Rundsch., 75:249-269.

Bird, M.I., Chivas, A.R., and Fyfe, W.S., 1991. Carbon ratios in the Amazon. Nature, 354:271-272.

Broecker, W.S., and Denton, G.H., 1989. The role of ocean-atmospheric reorganizations in glacial cycles. Geochim. Cosmochim. Acta, 53:24652501.

Cai, D.L., Tan, F.C., and Edmond, J.M., 1988. Sources and transport of particulate organic carbon in the Amazon river and estuary. Estuarine Coastal Shelf Sci., 26:1-14.

Charles, C.D., and Fairbanks, R.G., 1990. Glacial to interglacial changes in the isotopic gradients of Southern Ocean surface water. In Bleil, U., and Thiede, J. (Eds.), Proc. 1988 NATO Adv. Res. Workshop on Geological History of the Polar Oceans: Arctic versus Antarctic. NATO ASI Ser. C, 308:519-538

Charles, C.D., Wright, J.D., and Fairbanks, R.G., 1993. Thermodynamic influences on the marine carbon isotope record. Paleoceanography, 8:691-697.

Curry, W.B., and Crowley, T.J., 1987. The $\delta^{13} \mathrm{C}$ of equatorial Atlantic surface waters: implications for ice-age $\mathrm{pCO}_{2}$ levels. Paleoceanography, 2:489517.

Curry, W.B., Shackleton, N.J., Richter, C., et al., 1995. Proc. ODP, Init. Repts., 154: College Station, TX (Ocean Drilling Program).

Damuth, J.E., 1977. Late Quaternary sedimentation in the western equatorial Atlantic. Geol. Soc. Am. Bull., 88:695-710.

Damuth, J.E., and Kumar, N., 1975. Amazon Cone: morphology, sediments, age, and growth pattern. Geol. Soc. Am. Bull., 86:863-878.

Damuth, J.E., Flood, R.D., Kowsmann, R.O., Belderson, R.H., and Gorini, M.A., 1988. Anatomy and growth pattern of Amazon deep-sea fan as revealed by long-range side-scan sonar (GLORIA) and high-resolution seismic studies. AAPG Bull., 72:885-911.

Flood, R.D., Piper, D.J.W., and Shipboard Scientific Party, 1995. Introduction. In Flood, R.D., Piper, D.J.W., Klaus, A., et al., Proc. ODP, Init. Repts., 155: College Station, TX (Ocean Drilling Program), 5-16.

Flood, R.D., Piper, D.J.W., Klaus, A., et al., 1995. Proc. ODP, Init. Repts., 155: College Station, TX (Ocean Drilling Program).

Fontugne, M., and Duplessy, J.-C., 1986. Variations of the monsoon regime during the upper Quaternary: evidence from carbon isotopic record of organic matter in North Indian Ocean sediment cores. Paleogeogr., Paleoclimatol., Paleoecol., 56:69-88.

Hemleben, C., Spindler, M., and Anderson, O.R., 1989. Modern Planktonic Foraminifera: Berlin (Springer-Verlag).

Imbrie, J., Berger, A., Boyle, E., Clemens, S., Duffy, A., Howard, W., Kukla, G., Kutzbach, J., Martinson, D., McIntyre, A., Mix, A., Molfino, B., Morley, J., Peterson, L., Pisias, N., Prell, W., Raymo, M., Shackleton, N., and
Toggweiler, J., 1993. On the structure and origin of major glaciation cycles, 2. The 100,000-year cycle. Paleoceanography, 8:699-735.

Imbrie, J., Hays, J.D., Martinson, D.G., McIntyre, A., Mix, A.C., Morley, J.J., Pisias, N.G., Prell, W.L., and Shackleton, N.J., 1984. The orbital theory of Pleistocene climate: support from a revised chronology of the marine $\delta 180$ record. In Berger, A., Imbrie, J., Hays, J., Kukla, G., and Saltzman, B. (Eds.), Milankovitch and Climate (Pt. 1), NATO ASI Ser. C, Math Phys. Sci., 126: Dordrecht (D. Reidel), 269-305.

Jansen, J.H.F., Ufkes, E., and Schneider, R.R., 1996. Late Quaternary movements of the Angola-Benguela-Front, SE Atlantic, and implications for advection in the equatorial ocean. In Wefer, G., Berger, W.H., Siedler, G., and Webb, D. (Eds.), The South Atlantic: Present and Past Circulation: Berlin (Springer-Verlag), 553-575.

Kroon, D., 1988. The planktonic $\delta^{13} \mathrm{C}$ record, upwelling and climate. In Brummer, G.-J., Kroon, D. (Eds.), Planktonic Foraminifers as Tracers of Ocean-Climate History: Amsterdam (Free Univ. Press), 335-346.

Kroon, D., and Darling, K., 1995. Size and upwelling control of the stable isotope composition of Neogloboquadrina dutertrei (d'Orbigny), Globigerinoides ruber (d'Orbigny) and Globigerina bulloides d'Orbigny: examples from the Panama Basin and Arabian Sea. J. Foraminiferal Res., 25:39-52.

Kroon, D., and Ganssen, G., 1989. Northern Indian Ocean upwelling cells and the stable isotope composition of living planktonic foraminifers. Deep-Sea Res., 36:1219-1236.

Little, M.G., Kroon, D., Price, N.B., Schneider, R.R., Müller, P.J., and Wefer, G., in press. Late Quaternary changes in the Benguela upwelling system for the last 180,000 years. Palaeogeogr., Palaeoclimatol., Palaeoecol.

Manley, P.L., and Flood, R.D., 1988. Cyclic sediment deposition within Amazon deep-sea fan. AAPG Bull., 72:912-925.

Maslin, M., Mikkelsen, N., Burns, S., Sarnthein, M., 1995. Amazon fan high resolution stable isotope and faunal records of the last $30 \mathrm{ka}$; evidence for dramatic change: results from ODP Leg 155. Program and Abstracts ICP $V$, Halifax, 134. (Abstract)

Milliman, J.D., Summerhayes, C.P., and Barretto, H.T., 1975. Quaternary sedimentation on the Amazon continental margin: a model. Geol. Soc. Am. Bull., 86:610-614.

Müller, P.J., Schneider, R., and Ruhland, G., 1993. Late Quaternary $\mathrm{PCO}_{2}$ variations in the Angola Current: evidence from organic carbon $\delta^{13} \mathrm{C}$ and alkenone temperatures. In Zahn, R., Pedersen, T.F., Kaminski, M.A., and Labeyrie, L. (Eds.), Carbon Cycling in the Glacial Ocean: Constraints on the Oceanís Role in Gobal Change: NATO ASI Ser. I, 17: Heidelberg (Springer-Verlag), 343-366.

Oppo, D.W., and Fairbanks, R.G., 1989. Carbon isotope composition of tropical surface water during the past 22,000 years. Paleoceanography, 4:333-351.

Pastouret, L., Chamley, H., Delibrias, G., Duplessy, J.-C. and Thiede, J., 1978. Late Quaternary climatic changes in western tropical Africa deduced from deep-sea sedimentation off the Niger delta. Oceanol. Acta, $1: 217-232$.

Peterson, L.C., Lin, L.H., Murray, D.W., Overpeck, J.T., and Hughen K.A., 1995. Late Quaternary climate linkages between the tropical Atlantic/ Caribbean and far north Atlantic. Program and Abstracts ICP V, Halifax, p. 200. (Abstract)

Prell, W.L., Imbrie, J., Martinson, D.G., Morley, J.J., Pisias, N.G., Shackleton, N.J., and Streeter, H.F., 1986. Graphic correlation of oxygen isotope stratigraphy application to the late Quartenary. Paleoceanography, $1: 137-162$

Sarnthein, M., and Winn, K., 1990. Reconstruction of low and middle latitude export productivity, 30,000 years BP to Present: implications for global carbon reservoirs. In Schlesinger, S. (Ed.), Climate-Ocean Interaction: Dordrecht (Kluwer), 319-342.

Schneider, R., Dahmke, A., Kölling, A., Müller, P.J., Schulz, H.D., and Wefer, G., 1992. Strong deglacial minimum in the $\delta^{13} \mathrm{C}$ record from planktonic foraminifera in the Benguela upwelling region: palaeoceanographic signal or early diagenetic imprint. In Summerhayes, C.P., Prell, W.L., Emeis, K.C. (Eds.), Upwelling Systems: Evolution since the Early Miocene. Geol. Soc. Spec. Publ., 64:285-297.

Schneider, R.R., Müller, P.J., and Wefer, G., 1994. Late Quaternary paleoproductivity changes off the Congo deduced from stable carbon isotopes of planktonic foraminifera. Palaeogeogr., Palaeoclimatol., Palaeoecol., 110:255-274.

Schneider, R.R., Müller P.J., and Ruhland, G., 1995a. Late Quaternary surface circulation in the east equatorial Atlantic: evidence from alkenone sea surface temperatures. Paleoceanography, 10:197-219. 
Schneider, R.R., Müller, P.J., Price, B., Kroon, D., Alexander, I., 1995b. Late Quaternary Congo discharge fluctuations and influence of fluvial nutrient supply on paleoproductivity in the eastern Angola Basin. Program and Abstracts ICP V, Halifax, Canada, p. 137. (Abstract)

Schneider, R.R., Müller, P.J., Ruhland, G., Meinecke, G., Schmidt, H., and Wefer, G., 1996. Late Quaternary surface temperatures and productivity in the east-equatorial South Atlantic: response to changes in trade/monsoon wind forcing and surface water advection. In Wefer, G., Berger, W.H., Siedler, G., and Webb, D. (Eds.), The South Atlantic: Present and Past Circulation: Berlin (Springer-Verlag), 527-551.

Shackleton, N.J., 1977. Carbon-13 in Uvigerina: tropical rainforest history and the equatorial Pacific carbonate dissolution cycles. In Andersen, N.R., and Malahoff, A. (Eds.), The Fate of Fossil Fuel $\mathrm{CO}_{2}$ in the Oceans: New York (Plenum), 401-427.

Shackleton, N.J., Le, J., Mix, A.C., and Hall, M.A. 1992. Carbon isotope records from Pacific surface waters and atmospheric carbon dioxide. Quat. Sci. Rev., 11:387-400.

Showers, W.J., and Angle, D.G., 1986. Stable isotopic characterization of organic carbon accumulation on the Amazon continental shelf. Contin. Shelf Res., 6:227-244.

Showers, W.J., and Bevis, M., 1988. Amazon Cone isotopic stratigraphy: evidence for the source of the tropical freshwater spike. Palaeogeogr., Palaeoclimatol., Palaeoecol., 64:189-199.
Showers, W.J., and Margolis, S.V., 1985. Evidence for a tropical freshwater spike during the last glacial/interglacial transition in the Venezuela Basin: $\delta^{18} \mathrm{O}$ and $\delta^{13} \mathrm{C}$ of calcareous plankton. Mar. Geol., 68:145-165.

Showers, W.J., Genna, B., and Price, P., 1995. Amazon continental margin high resolution records of western tropical Atlantic circulation over the past 130 ky. Program and Abstracts ICP V, Halifax, p. 139. (Abstract)

Summerhayes, C.P., Kroon, D., Rosell-Melé, A., Jordan, R.W., Schrader, HJ., Hearn, R., Villanueva, J., Grimalt, J.O., and Eglinton, G., 1995. Variability in the Benguela Current upwelling system over the past 70,000 years. Prog. Oceanogr., 35:207-251.

Westerhausen, L., Poynter, J., Eglinton, G., Erlenkeuser, H., and Sarnthein, M., 1993. Marine and terrigenous origin of organic matter in modern sediments of the equatorial East Atlantic: the $\delta^{13} \mathrm{C}$ and molecular record. Deep-Sea Res., 140:1087-1121.

Date of initial receipt: 5 December 1995

Date of acceptance: 6 June 1996

Ms 155SR-228 